\title{
Longitudinal instabilities in an air-blasted liquid sheet
}

\author{
By ANTONIO LOZANO1†, FÉLIX BARRERAS ${ }^{2}$ \\ GUILLERMO HAUKE ${ }^{2}$ AND CÉSAR DOPAZO \\ ${ }^{1}$ LITEC/CSIC, Maria de Luna, 3, 50015-Zaragoza, Spain \\ ${ }^{2}$ Centro Politécnico Superior de Ingenieros, Area de Mecánica de Fluidos, \\ Universidad de Zaragoza, Maria de Luna, 3, 50015-Zaragoza, Spain
}

(Received 7 March 2000 and in revised form 4 December 2000)

An experimental and numerical study has been performed to improve the understanding of the air/liquid interaction in an air-blasted breaking water sheet. This research is focused in the near field close to the exit slit, because it is in this region where instabilities develop and grow, leading to the sheet breakup. In the experiments, several relevant parameters were measured including the sheet oscillation frequency and wavelength, as well as the droplet size distribution and the amplification growth rate. The flow was also investigated using linear instability theory. In the context of existing papers on instability analysis, the numerical part of this work presents two unique features. First, the air boundary layer is taken into account, and the effects of air and liquid viscosity are revealed. Second, the equations are solved for the same parameter values as those in the experiments, enabling a direct comparison between calculations and measurements; although qualitatively the behaviour of the measured variables is properly described, quantitative agreement is not satisfactory. Limitations of the instability analysis in describing this problem are discussed. From all the collected data, it is confirmed that the oscillation frequency strongly depends on the air speed due to the near-nozzle air/water interaction. The wave propagates with accelerating interface velocity which in our study ranges between the velocity of the water and twice that value, depending on the air velocity. For a fixed water velocity, the oscillation frequency varies linearly with the air velocity. This behaviour can only be explained if the air boundary layer is considered.

\section{Introduction}

Despite the importance of spray flows in many daily applications, the basic physical mechanisms that cause the atomization processes are still not completely understood. A common type of atomizer, used for example in aircraft turbines, is the so-called 'air-blast', in which a high-speed air stream surrounds a liquid jet. Although most air-blast commercial atomizers have an axisymmetric design, two-dimensional geometries where the liquid is injected as a thin planar sheet are becoming increasingly popular as a benchmark flow to study primary atomization because of its relative experimental simplicity. The basic mechanisms leading to the liquid breakup are the same in both axisymmetric and planar configurations, but planar designs are easier to visualize. Large-aspect-ratio sheets are especially suitable for studying the transverse 
perturbations in regions near the nozzle exit, and edge effects in the injection region are limited to a small fraction of the spanwise dimension.

Studies on the breakup of a liquid mass into droplets to generate a spray, initiated in a systematic way in the past century, are very extensive. Restricting our consideration to those that directly refer to the present geometry, the first reported work on the disintegration of a liquid sheet known to the authors is a series of experiments by Felix Savart in 1833. In this case, the sheet was formed by impinging a water jet onto a disk, forming a water bell similar to those studied a century later by Taylor (1959a). Over the last five decades, experimental studies, mostly based on visual observations, have progressed together with theoretical stability analysis. Squire (1953), York, Stubbs \& Tek (1953), Taylor (1959b) and Hagerty \& Shea (1955) initiated linear stability analysis of thin liquid sheets in quiescent air, considering potential flows. Sinusoidal (antisymmetric) and varicose (dilatational or symmetric) waves were predicted. Liquidfilm experimental disintegration studies in high-aspect-ratio two-dimensional designs were initiated by Hagerty \& Shea (1955) although in their experiments, water was also exiting into non-flowing ambient air. In that report, initial perturbations were introduced by oscillating the nozzle. The two types of waves predicted, sinusoidal and varicose, were observed at the liquid interface.

Research on liquid sheets was continued, although in slightly different geometries, with the work of Taylor (1959a) on water bells, and the numerous papers, both experimental and theoretical, by Dombrowski and coworkers on short-aspect-ratio nozzles that produced the so-called water fans (Dombrowski, Hasson \& Ward 1960; Fraser, Dombrowski \& Routley 1963). Dombrowski \& Johns (1963) assumed a viscous liquid sheet in a quiescent inviscid gas. From a balance of the forces acting upon a fluid element at the interface they concluded that liquid viscosity added a dependence of the growth rate not only on wavenumber but also on sheet thickness. Crapper, Dombrowski \& Pyott (1975b) unsuccessfully tried to compare their inviscid system results to experimental data. In all these investigations, boundary conditions at the gas/liquid interfaces corresponded to those of a shear-free surface. With this simplification, the tangential stress at the liquid was set equal to zero and continuity of the perturbed velocity was not required. The gas dynamic effect was, thus, completely ignored. To describe a more realistic configuration, Crapper, Dombrowski \& Jepson $(1975 a)$ considered viscosity in both fluids, a slightly parabolic velocity profile for the basic liquid flow and an exponentially decaying profile for the air stream. When solving the equations, the parabolic water profile was further approximated by a straight line. The frequency instability range was widened due to viscosity and, for high liquid velocities, no maxima for the growth rate curve as a function of the wavenumber were predicted when surface tension was taken into account. It was also found that the instabilities were insensitive to changes in liquid viscosity.

Experiments in large-aspect-ratio sheets were resumed years later, when Rizk \& Lefebvre (1980), Arai \& Hashimoto (1985), Mansour \& Chigier (1990, 1991) and Stapper \& Samuelsen (1990) sandwiched the liquid sheet between two high-speed coflowing air streams to produce for the first time an air-blast atomization. Most of these studies, based on visual observations, were phenomenological, with the main interest in predicting the droplet cloud characteristics. Correlations were obtained relating physical fluid properties, nozzle geometry and flow conditions with mean droplet diameter, breakup length and oscillation frequency. It was concluded that the oscillation frequency is linearly dependent on air velocity, and only weakly dependent on the liquid one. For low gas/liquid velocity ratios a mixture of dilatational and sinusoidal waves was observed, with small growth rates. It was also 
detected that on increasing the velocity ratio, the sinusoidal mode became dominant and amplification was highly enhanced. A transition in breakup characteristics is described, passing from what Stapper \& Samuelsen denoted as a 'cellular' mode to a 'stretched streamwise ligament' mode in which ligaments oriented parallel to the flow direction are formed prior to disintegration. Effects of surface tension and liquid viscosity were analysed in the measurements by Eroglu \& Chigier (1991), Stapper, Sowa \& Samuelson (1992) and Shavit \& Chigier (1995). The main conclusion was that these properties were affecting the characteristic length and time scales associated with the breakup, and consequently were influential on the mean droplet diameter. The breakup mechanisms are, however, largely independent of them.

From a numerical point of view, Lin, Lian \& Creighton (1990) found that a viscous ambient gas dragged by the liquid motion tends to destabilize the sheet and to shorten both sinuous and varicose mode wavelengths. Li \& Tankin (1991) treated a viscous liquid sheet in an inviscid gas and conducted a temporal growth study, obtaining a viscosity-enhanced instability the same as that found by Crapper et al. (1975a). Rangel \& Sirignano (1991) performed linear and nonlinear stability analysis of a liquid sheet in a moving gas, both considered to be inviscid. Ibrahim (1994) analysed the spatial growth of a perturbation for the same system as that proposed by Li \& Tankin, obtaining different results. These studies were extended to a three-dimensional configuration in Ibrahim \& Akpan (1996).

Conditions where the liquid sheet exits into a quiescent atmosphere are intended to describe those of pressure atomizers. When predictions obtained in studies describing this situation are directly applied to air-blasted geometries, comparisons with experimental results fail dramatically, because the sheet oscillation has been proven to be mainly controlled by the air flow dynamics (Barreras 1998). The problem in the context of an air-blasted configuration has been treated, after Rangel \& Sirignano (1991), by Yang (1992), Ibrahim (1995) and Cousin \& Dumouchel (1996), with the air exit velocity higher than that of the liquid. None of these papers, however, has included air viscosity in the perturbation equations.

The only papers where a viscous boundary-layer-type velocity profile has been considered for the basic state of the gas stream describe simpler physical situations. Teng, Lin \& Chen (1997) numerically investigated two semi-infinite viscous fluids with parabolic velocity profiles as the basic states and used Chebyshev polynomial expansions to solve the Orr-Sommerfeld equation and boundary conditions. Absolute instability did occur when the Weber number was approximately one, surface tension effects being the cause. Interfacial shear plays a crucial role in other related problems, such as wind-generated waves (Benjamin 1959; Miles 1962) and wind-sheared liquid films in contact with solid walls (Smith \& Davis 1982; Yih 1990; Miesen \& Boërsma 1995; Criminale et al. 1997).

Recent experimental works have emphasized the analysis of the near-field region, close to the exit slit, to explain the development and growth of the instabilities that ultimately cause the liquid sheet breakup (Lozano et al. 1996; Vich, Dumouchel \& Ledoux 1996). In order to explain the fast amplification of initial perturbations it has been suggested (Vich et al.) that some mechanism other than the Kelvin-Helmholtz instability could be contributing to enhance their growth, for example, the 'flag effect' described by Birkhoff (1962).

This paper presents a combined experimental and numerical investigation of a large-aspect-ratio air-blasted liquid sheet, aimed at improving the understanding of the air/liquid interaction in the region near the nozzle exit. It is in this region where 
instabilities are triggered, develop and grow, leading to the sheet breakup. In the experimental part of the study, different parameters have been examined, applying a variety of techniques. In particular, measurements have been made of the sheet oscillation frequency, droplet size distribution and growth rate, and estimations have been obtained of the wavelength and wave propagation speed.

The flow has also been analysed using linear instability theory, reproducing the conditions of the experiments. In this way, comparison and validation of the numerical predictions becomes almost immediate. The present analysis is also novel in that, unlike most of the previously published studies, the air boundary layer has been taken into account, and the effects of air and liquid viscosity have been considered. Air and liquid basic flows have been taken as viscous, as have their perturbations. In doing so, the interface boundary conditions better reflect the real situation.

\section{Description of the experiment}

The characteristics of the experimental set up used in the present study are similar to those described by Mansour \& Chigier (1990) and Lozano et al. (1996), with one of the main differences being both water and air nozzle profiles, which were carefully designed so that the air velocity at the exit is parallel to the water flow. A schematic of the atomizer assembly is depicted in figure 1 together with a photograph detailing the nozzle head. Water injected at the top of the nozzle head exits vertically through a $0.35 \mathrm{~mm}$ wide slit. The nozzle was contoured, fitting a sixth-order polynomial, to ensure a uniform velocity profile and has a $23: 1$ contraction ratio. The span of the sheet is $80 \mathrm{~mm}$, yielding an aspect ratio of 230 . The edge thickness of the nozzle lips is $0.35 \mathrm{~mm}$. Air is also introduced from the top following a settling chamber with two honeycombs and a wire mesh screen to smooth the flow. The air channels located at both sides of the liquid nozzle were also contoured according to sixthorder polynomials, with contraction ratios of $15: 1$ and exit widths of $3.45 \mathrm{~mm}$. This geometry probably does not provide the most efficient atomization, as air impinging at an angle with respect to the liquid sheet would probably produce a faster breakup. Its simplicity, however, is an advantage in order to compare the measurements with numerical simulations and identify basic breakup mechanisms. For the conditions under study, water velocities ranged from 0.6 to $6 \mathrm{~m} \mathrm{~s}^{-1}$, while air velocities varied between 15 and $75 \mathrm{~m} \mathrm{~s}^{-1}$.

The sheet oscillation frequency was measured using the laser diffraction technique described in detail in Mansour \& Chigier (1991). A beam generated by a $5 \mathrm{~mW}$ diode laser propagates horizontally parallel to the liquid sheet, pointing directly to a receiving photodiode. The beam is slightly displaced from the nozzle slit. As the sheet oscillates, the liquid/water interface cuts the light beam, reflecting and refracting part of it, and its passage is detected as a drop in the luminous signal registered by the photodiode. The periodic signal from the photodiode output is digitized, and the fundamental frequency is obtained from a Fourier transform. As shown in Mansour \& Chigier (1991) and in Lozano et al. (1996) for a wide range of combinations of air and water velocities, the photodiode signal clearly presents a dominant oscillation frequency, which is easily discernible in the transformed domain. As also explained in the cited references, the signal is largely independent of the downstream position of the photodiode, provided that it is not located inside the spray region.

Droplet size distributions were measured using a Malvern 2600 laser difractometer. The measurements were obtained at $1 \mathrm{~cm}$ from the limit of the intact sheet distance, to minimize effects of secondary breakup and droplet coalescence. To determine the 

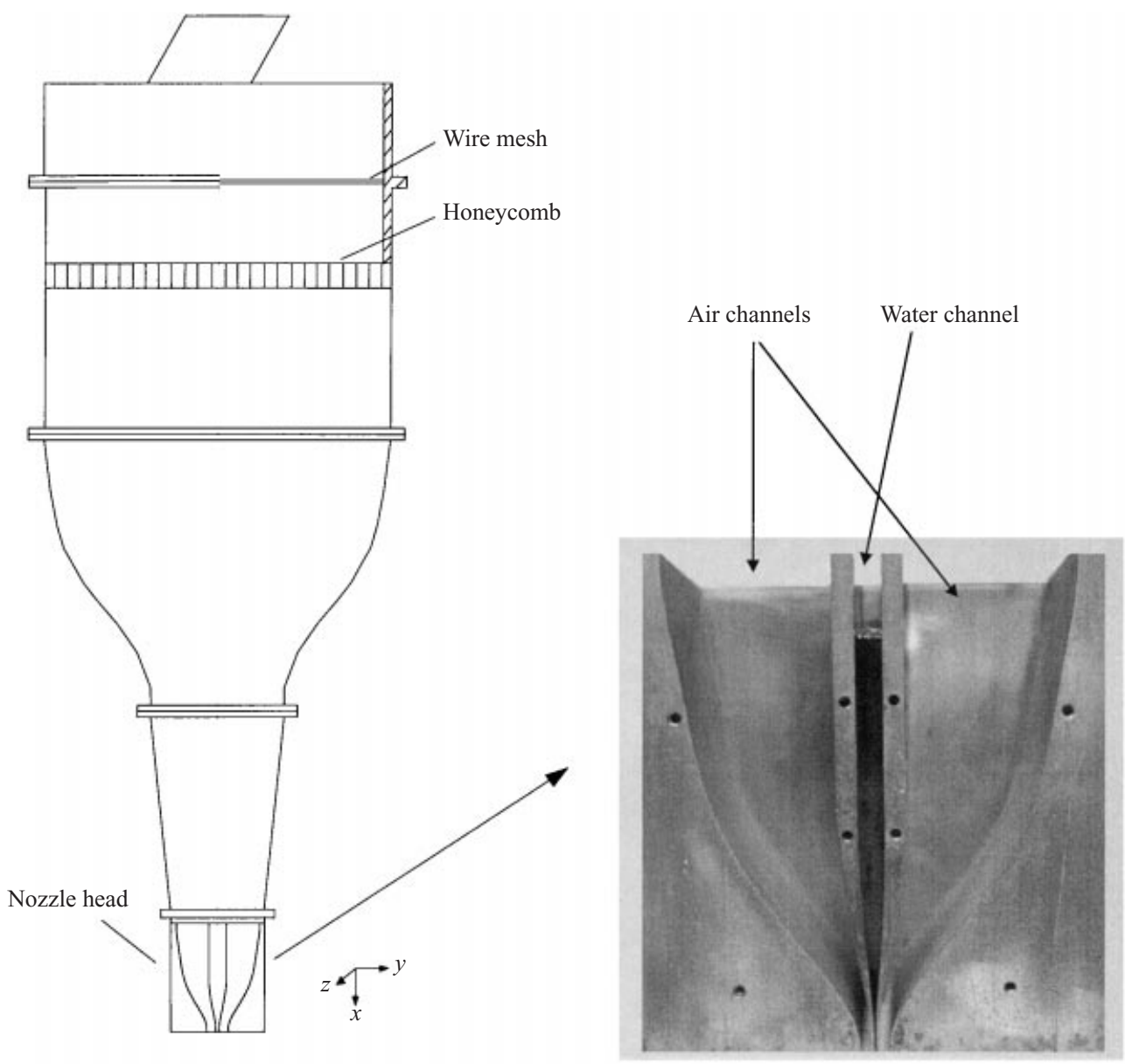

FiguRE 1. View of the atomizer assembly and the nozzle head.

average diameter, 1000 samples were collected for each flow configuration, characterized by the air and water velocity values. From the size distributions, the Sauter mean diameter ( $S M D$ or $D_{32}$ ) was obtained, according to the following expression:

$$
S M D=\frac{\int_{0}^{\infty} D^{3} F(D) \mathrm{d} D}{\int_{0}^{\infty} D^{2} F(D) \mathrm{d} D},
$$

where $D$ is the droplet diameter and $F(D)$ is the size distribution function (see, for example, Lefebvre 1989).

To study the wavelength and growth rate of the longitudinal perturbation, planar laser-induced fluorescence (PLIF) images were obtained, seeding the water with a small concentration of sulphorhodamine B (Kiton Red). To excite the dye, a doublecavity Quantel YG781C-10 pulsed Nd:YAG laser was used, doubling the frequency of its emission to obtain $100 \mathrm{~mJ}$ pulses (out of a maximum energy of $500 \mathrm{~mJ}$ ) at $532 \mathrm{~nm}$ with a pulse duration of $6 \mathrm{~ns}$. The laser was formed into a sheet $5 \mathrm{~cm}$ high and $500 \mu \mathrm{m}$ thick. The absorption spectrum of sulphorhodamine B has a maximum at $556 \mathrm{~nm}$, hence excitation at $532 \mathrm{~nm}$ is very efficient (Brackmann 1994). The fluorescence peak 


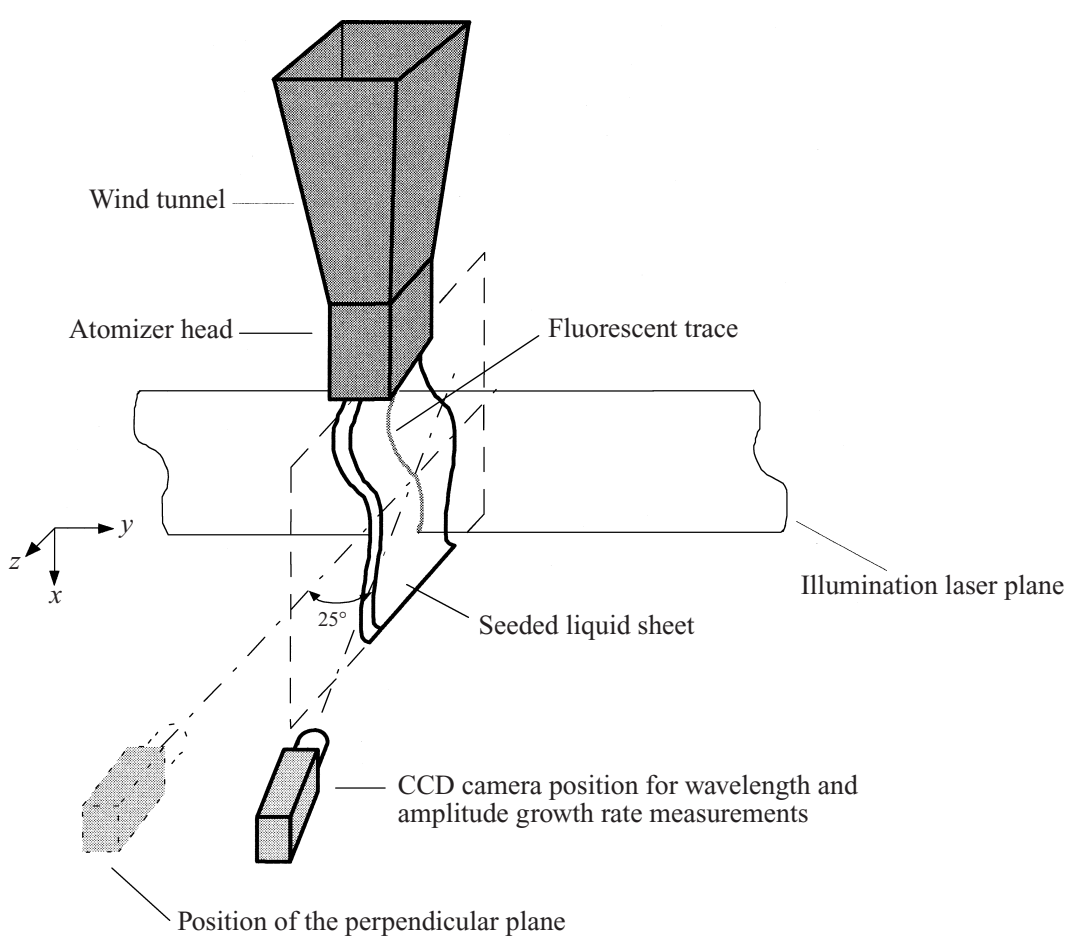

FIGURE 2. Schematic of the image acquisition configuration for the fluorescence measurements.

of this dye is located at $575 \mathrm{~nm}$, which allows efficient discrimination between the emitted signal and the excitation source. In these experiments, a $6 \mathrm{~mm}$ thick Schott OG 550 filter was placed in front of the camera lens, to reject the strong $532 \mathrm{~nm}$ Mie scattering from the liquid droplets.

To image the fluorescence emission, a Princeton Instruments slow-scan CCD camera was used, with a $50 \mathrm{~mm}$ F1.2 Nikon lens. It should be noted that the short lifetime of the fluorescence enables high-temporal-resolution imaging without the need of gated cameras. Images were acquired for longitudinal sections slicing the water sheet perpendicularly through the middle of the exit slit. Data sets were recorded for two different fields of view, $26 \times 35 \mathrm{~mm}$ with a resolution of $90 \mu \mathrm{m} /$ pixel and $13 \times 17.5 \mathrm{~mm}$ with a resolution of $45 \mu \mathrm{m} /$ pixel. To avoid imaging through the sheet edge, thickened by surface tension, the camera was not located perpendicular to the liquid sheet, but at an angle of $25^{\circ}$ (see figure 2). This arrangement introduces a perspective deformation, which has been neglected because it has been calculated to produce a length distortion of less than $1 \%$. It also causes a deformation in the coordinate perpendicular to the sheet due to the fact that a rotated plane is effectively imaged, thus shortening the location of the points in the air/water interface by $11 \%$. This effect does not affect the wavelength measurements, but it has been taken into account and corrected in the measurements of the growth rate.

Wavelengths were measured from instantaneous PLIF images. To measure the oscillation-amplitude growth rate, mean images were obtained averaging on-chip

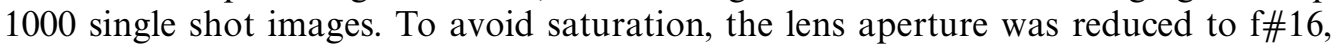
as well as the laser energy. To determine the interface location the images were thresholded and binarized. 


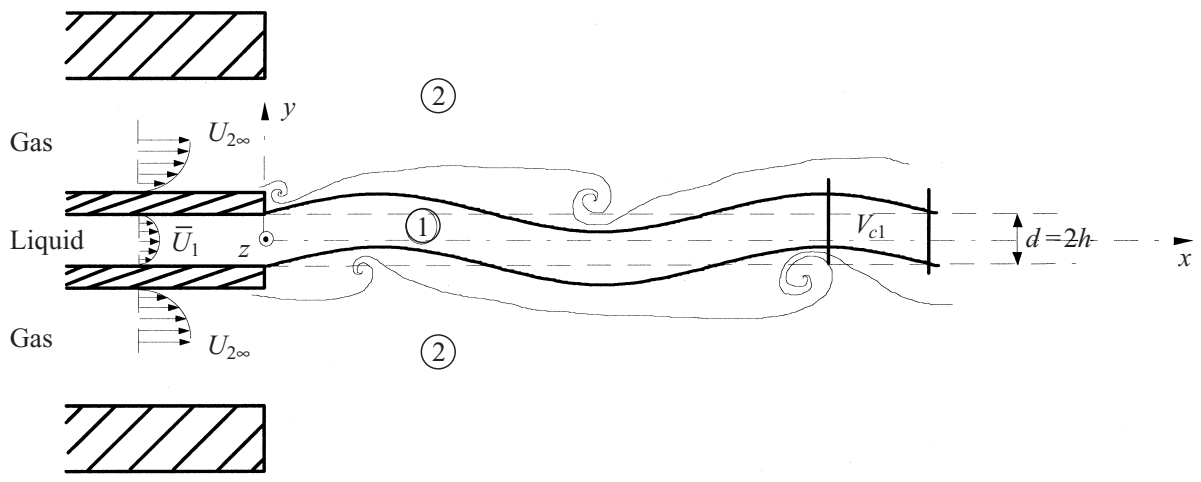

FIGURE 3. Physical problem under study. Sketch of the flow.

\section{Modelling formulation}

A thin liquid sheet sheared by a high-speed gas, downstream of the trailing edges of two parallel plates, as sketched in figure 3 is considered. In the neighbourhood of the plate edges, the basic velocity profiles start their transition from non-slip boundary conditions on the plate surfaces to a moving gas/liquid interface. At high gas velocities, vortex shedding will occur at the corners of the trailing edge. These vortices will induce perturbations of the velocity and pressure fields at both gas/liquid interfaces. The action of the gas upon the liquid sheet can be expressed as a surface integral over the interfaces, $S_{I}$, namely

$$
\boldsymbol{F}_{G / L}=\int_{S_{I}}\left(-p_{2} \hat{\boldsymbol{n}}+\tau_{2}^{\prime} \cdot \hat{\boldsymbol{n}}+\sigma K \hat{\boldsymbol{n}}\right) \mathrm{d} S,
$$

where $p_{2}$ is the gas pressure, $\tau_{2}^{\prime}$ is the gas viscous stress tensor, $\sigma$ is the gas/liquid surface tension, $K$ is the interface main curvature and $\hat{\boldsymbol{n}}$ is the interface unit normal vector pointing towards the gas.

Making the perturbed integral momentum equation non-dimensional by dividing by the inertia forces, the following dimensionless groups emerge:

Reynolds numbers

Momentum flux ratio

$$
R e_{1}=\frac{U_{1} h}{v_{1}}, \quad R e_{2}=\frac{U_{2} \delta}{v_{2}},
$$

$$
M F R=\frac{\rho_{2} U_{2}^{2}}{\rho_{1} U_{1}^{2}}
$$

$$
W e=\frac{\rho_{1} U_{1}^{2} h}{\sigma},
$$

where $2 h$ is the sheet thickness, and $\delta$ may be taken as the air boundary layer thickness.

Under certain conditions, the sheet oscillates with a dominant frequency. In this case, the oscillation can be characterized by a Strouhal number, $S t$, using a velocity and a length scale to non-dimensionalize the frequency. As will be seen in the following paragraphs, the sheet thickness, $d=2 h$, and the air velocity, $U_{2}$, are suitable parameters for this purpose because the resulting Strouhal number is approximately constant, and with this definition: 


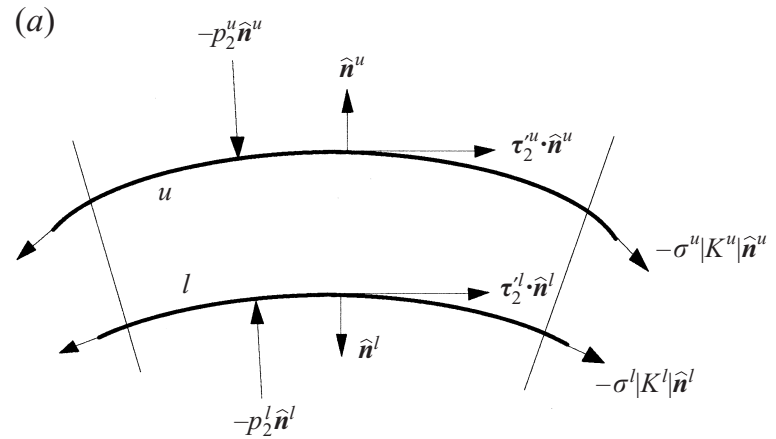

(b)

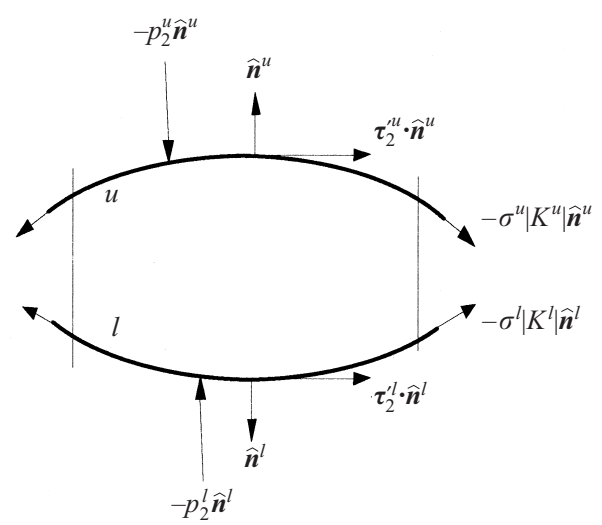

FIGURE 4. Force diagram over a liquid sheet element. (a) Antisymmetric waves. (b) Symmetric case.

Strouhal number

$$
S t=f d / U_{2} \text {. }
$$

For the present experiments $h, v_{1}, v_{2}, \rho_{1}, \rho_{2}$ and $\sigma$ are given. The sheet oscillation frequency, $f_{1}$, and $\delta$ will vary as a consequence of changing the liquid and gas velocities, $U_{1}$ and $U_{2}$ respectively.

The balance of pressure, viscous and surface tension forces, integrated over the interfaces, determines the motion of an element of the liquid sheet. For an antisymmetric wave the area difference of the upper and lower interface elements, denoted by superindices $u$ and $l$ respectively in figure $4(a)$, together with asymmetries in the pressure and surface tension forces, will yield a resultant force with a component normal to the sheet that will induce flapping. Viscous stresses cause a tangential resultant force that will deform the sheet in all directions. For a symmetric wave, figure $4(b)$, symmetric pressure distributions and surface tension do not induce oscillations. On the contrary, they play a stabilizing effect inhibiting the amplitude growth. It is for this reason that atomization of the liquid sheet always occurs as a consequence of the growth of a sinusoidal oscillation, as observed in the experiments. The growth of sinusoidal waves has been studied by means of linear instability analysis.

As is well known, linear perturbation analysis is based on perturbing a steady-state solution of the flow with a small-amplitude wave in normal modes, analysing its growth rate with the linearized Navier-Stokes equations. The wave amplitude has to be small compared with its wavelength or with the sheet thickness. A simplified steadystate solution is usually assumed, e.g. considering quiescent air, neglecting its shearing 


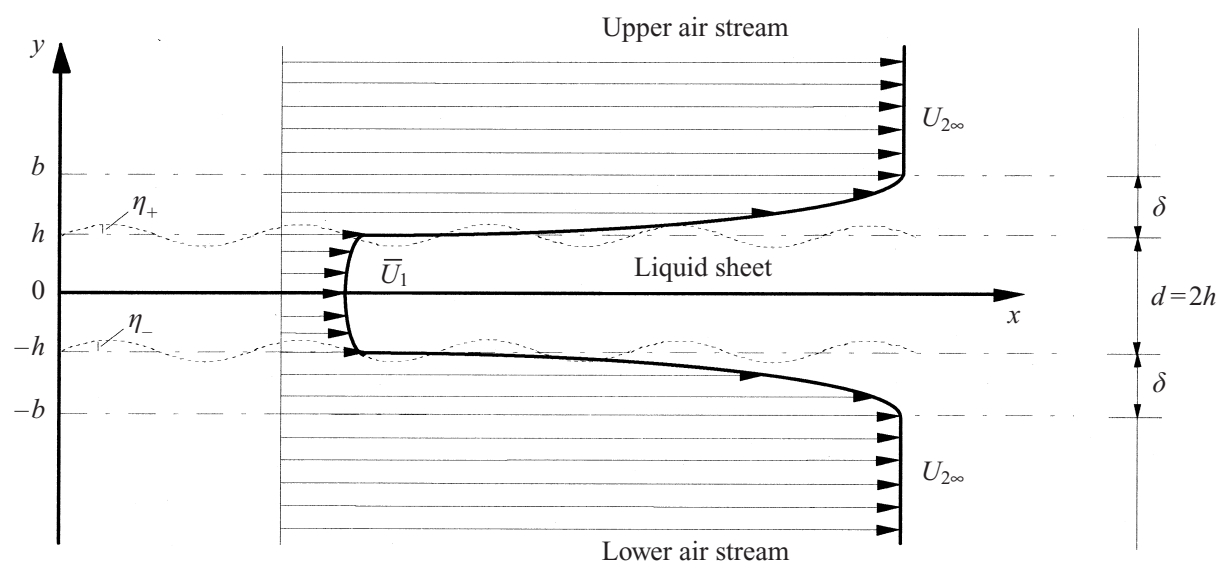

FIGURE 5. Sketch of the flow configuration considered for the linear instability analysis.

effects (zero viscosity), constant water velocity profile, etc. These simplifications could imply some inconsistencies in the boundary conditions at the interfaces that are normally ignored. The instability temporal growth is, usually, analysed in an infinite sheet.

In the numerical part of the present study, the linear instability analysis of a two-dimensional sheet of constant thickness, $2 h$, of a viscous liquid (water) sandwiched between two identical semi-infinite viscous air streams was considered. HighReynolds-number air flows were assumed, thus characterizing them by the speed $U_{2 \infty}$ far from the liquid sheet centreline and by the local air boundary layer thickness, $\delta$. The basic velocity profiles for the water sheet and the air boundary layer were approximated by quadratic functions of $y$, independent of the downstream coordinate (see figure 5). The unperturbed velocity profiles were, therefore, approximated by

$$
\begin{gathered}
\hat{U}_{1}(y)=a_{0}+a_{2} y^{2}, \quad-h \leqslant y \leqslant h, \\
\hat{U}_{2}(y)=b_{0}+b_{1}\left(\frac{y-h}{\delta}\right)+b_{2}\left(\frac{y-h}{\delta}\right)^{2}, \quad h \leqslant y \leqslant h+\delta, \\
\hat{U}_{2}(y)=b_{0}-b_{1}\left(\frac{y+h}{\delta}\right)+b_{2}\left(\frac{y-h}{\delta}\right)^{2}, \quad-(h+\delta) \leqslant y \leqslant-h,
\end{gathered}
$$

with $h \leqslant y \leqslant h+\delta$ for the upper air stream and $-(h+\delta) \leqslant y \leqslant-h$ for the lower air stream. The unknown coefficients $a_{i}$ and $b_{i}$ are calculated by imposing the following five constraints:

(i) the volumetric water flow rate or, equivalently, the liquid mean velocity, $\bar{U}_{1}$, is given;

(ii) the air and liquid velocities at the unperturbed interfaces, $y= \pm h$, are equal;

(iii) the tangential viscous stresses balance at the unperturbed interface;

(iv) air velocities at the boundary layer edges, $y= \pm(h+\delta)$ are equal to $U_{2 \infty}$;

(v) the air velocity gradients at the boundary layer edges vanish.

Solving the five algebraic equations yields

$$
a_{0}=\bar{U}_{1}\left(1+\frac{1}{3} \frac{\mu_{r}}{\Delta}\right)-U_{2 \infty} \frac{1}{3} \frac{\mu_{r}}{4},
$$




$$
\begin{gathered}
a_{2}=\frac{U_{2 \infty}-\bar{U}_{1}}{h^{2}} \frac{\mu_{r}}{\Delta}, \\
b_{0}=U_{2 \infty}\left(1-\frac{l}{\Delta}\right)+\bar{U}_{1} \frac{l}{\Delta}, \\
b_{1}=\left(U_{2 \infty}-\bar{U}_{1}\right) \frac{2 l}{\Delta}, \\
b_{2}=-\left(U_{2 \infty}-\bar{U}_{1}\right) \frac{l}{\Delta},
\end{gathered}
$$

where $\mu_{r}=\mu_{2} / \mu_{1}$ is the air/water viscosity ratio, $l=\delta / h$ is the ratio of the air boundary layer thickness to the liquid sheet half-thickness and $\Delta=(2 / 3) \mu_{r}+l$.

The linearized equations governing this problem are

$$
\begin{gathered}
\frac{\partial u_{\alpha}}{\partial x}+\frac{\partial v_{\alpha}}{\partial y}=0, \\
\frac{\partial u_{\alpha}}{\partial t}+\hat{U}_{\alpha} \frac{\partial u_{\alpha}}{\partial x}+v_{1} \frac{\partial \hat{U}_{\alpha}}{\partial y}=-\frac{1}{\rho_{\alpha}} \frac{\partial p_{\alpha}}{\partial x}+v_{\alpha}\left(\frac{\partial^{2} u_{\alpha}}{\partial x^{2}}+\frac{\partial^{2} v_{\alpha}}{\partial y^{2}}\right), \\
\frac{\partial v_{\alpha}}{\partial t}+\hat{U}_{\alpha} \frac{\partial v_{\alpha}}{\partial x}=-\frac{1}{\rho_{\alpha}} \frac{\partial p_{\alpha}}{\partial y}+v_{\alpha}\left(\frac{\partial^{2} u_{\alpha}}{\partial x^{2}}+\frac{\partial^{2} v_{\alpha}}{\partial y^{2}}\right),
\end{gathered}
$$

where $\alpha=1,2$ represents liquid or gas phase, respectively.

The appropriate boundary conditions are velocity continuity and stress balance across the interface plus the kinematic condition, derived from considering the equation of the interface $S(x, y, t)=\eta_{ \pm}(x, t)-y=0$, where $\eta_{+}(x, t)$ and $\eta_{-}(x, t)$ are the upper and lower interface displacements from the equilibrium position. For sinuous waves $\eta_{+}(x, t)=\eta_{-}(x, t)$, while for varicose waves $\eta_{+}(x, t)=-\eta_{-}(x, t)$. As previously explained, experimental observations indicate that under the most efficient atomization conditions, the sheet oscillates in a dominant sinusoidal mode. For this reason, in the present calculations only sinusoidal waves were considered.

The boundary conditions at the interface $y= \pm h+\eta_{+}(x, t)$ were linearized about $y= \pm h$ and only the first-order perturbations were retained. This results in the following equations:

continuity of $u$

continuity of $v$

$$
u_{1}( \pm h)+\eta_{ \pm} \frac{\mathrm{d} \hat{U}_{1}( \pm h)}{\mathrm{d} y}=u_{2}( \pm h)+\eta_{ \pm} \frac{\mathrm{d} \hat{U}_{2}( \pm h)}{\mathrm{d} y}
$$

$$
v_{1}( \pm h)=v_{2}( \pm h)
$$

continuity of tangential stress

$$
\mu_{1}\left(\frac{\partial u_{1}}{\partial y}+\frac{\partial v_{1}}{\partial x}\right)=\mu_{2}\left(\frac{\partial u_{2}}{\partial y}+\frac{\partial v_{2}}{\partial x}\right),
$$

normal stress jump

kinematic condition

$$
p_{1}-2 \mu_{1} \frac{\partial v_{1}}{\partial y}=p_{2}-2 \mu_{2} \frac{\partial v_{2}}{\partial y}-\sigma \frac{\partial^{2} \eta}{\partial x^{2}}
$$

$$
v_{1}=\frac{\partial \eta}{\partial t}+\hat{U}_{1} \frac{\partial \eta}{\partial x} .
$$


Note that the gradient $\mathrm{d} \hat{U}_{\alpha} / \mathrm{d} y$ is not continuous across the air/water interface. For this reason, continuity of $u$ cannot be simply approximated by $u_{1}( \pm h)=u_{2}( \pm h)$. A complete discussion about this point can be found in Yih (1990).

In order to match the solution of the problem for the air boundary layers with the region of the free stream with constant velocity $U_{2 \infty}$, certain boundary conditions established at virtual interfaces, located at $y= \pm b$ where $b=h+\delta$ must be satisfied. These conditions are

continuity of $u$

$$
u_{2}( \pm b)+\eta_{b} \frac{\mathrm{d} \hat{U}_{2}( \pm b)}{\mathrm{d} y}=u_{2}^{*}( \pm b)
$$

continuity of $v$

$$
v_{2}( \pm b)=v_{2}^{*}( \pm b)
$$

continuity of tangential stress

$$
\left(\frac{\partial u_{2}}{\partial y}+\frac{\partial v_{2}}{\partial x}\right)=\left(\frac{\partial u_{2}^{*}}{\partial y}+\frac{\partial v_{2}^{*}}{\partial x}\right)
$$

continuity of normal stress

$$
p_{2}-2 \mu_{2} \frac{\partial v_{2}}{\partial y}=p_{2}^{*}-2 \mu_{2} \frac{\partial v_{2}^{*}}{\partial y}
$$

kinematic condition

$$
v_{2}=\frac{\partial \eta_{b}}{\partial t}+\hat{U}_{2} \frac{\partial \eta_{b}}{\partial x}
$$

where the asterisk denotes variables corresponding to the free-stream region. To these conditions, there is yet to add vanishing perturbation velocity far from the centreline,

$$
u_{2}^{*}( \pm \infty)=v_{2}^{*}( \pm \infty)=0 .
$$

This viscous two-dimensional problem can be conveniently solved in terms of the stream function, $\psi(x, y, t)$, formulation. Expressing the perturbed functions in a normal mode decomposition:

$$
[\psi(x, y, t), p(x, y, t), \eta(x, t)]=\left[\Psi(y), P(y), \eta_{0}\right] \exp (\mathrm{i} k x+\omega t) .
$$

Before proceeding, the variables are transformed into a non-dimensional form, using appropriate velocity and length scales for both air and liquid flows, yielding

$$
\left.\begin{array}{l}
\tilde{y}=\frac{y}{h}, \quad \tilde{\omega}=\frac{\omega h}{\bar{U}_{1}}, \quad \tilde{k}=k h, \quad \tilde{\eta}_{ \pm}=\frac{\eta_{ \pm}}{h}, \\
\tilde{U}_{1}(\tilde{y})=\frac{\hat{U}_{1}(y)}{\bar{U}_{1}}, \quad \tilde{\psi}_{1}(\tilde{y})=\frac{\psi_{1}(y)}{\bar{U}_{1} h}, \quad \tilde{U}_{2}(\tilde{y})=\frac{\hat{U}_{2}(y)}{U_{2 \infty}}, \quad \tilde{\psi}_{2}(\tilde{y})=\frac{\psi_{2}(y)}{U_{2 \infty} \delta} .
\end{array}\right\}
$$

This non-dimensional analysis transforms the liquid domain to $-1 \leqslant \tilde{y} \leqslant 1$, the upper gas domain to $1 \leqslant \tilde{y} \leqslant 1+l$ and the lower gas domain to $-(1+l) \leqslant \tilde{y} \leqslant-1$, where $l=\delta / h$. In this way, the final Orr-Sommerfeld equations to solve are

$$
\begin{aligned}
& \frac{1}{R e_{1}} \tilde{\Psi}_{1}^{\mathrm{iv}}(\tilde{y})-\left[\omega+\mathrm{i} k \tilde{U}_{1}(\tilde{y})+\frac{2}{R e_{1}}\right] \tilde{\Psi}_{1}^{\prime \prime}(\tilde{y}) \\
& +\left[k^{2}\left(\omega+\mathrm{i} k \tilde{U}_{1}(\tilde{y})+\frac{k^{2}}{\operatorname{Re} e_{1}}\right)+\mathrm{i} k \tilde{U}_{1}^{\prime \prime}(\tilde{y})\right] \tilde{\Psi}_{1}(\tilde{y})=0,
\end{aligned}
$$




$$
\begin{aligned}
\frac{1}{R e_{2}} \tilde{\Psi}_{2}^{\mathrm{iv}}(\tilde{y})-\left[\omega V_{12}+\mathrm{i} k \tilde{U}_{2}(\tilde{y})+\frac{2 l}{R e_{2}}\right] \tilde{\Psi}_{2}^{\prime \prime}(\tilde{y}) \\
+\left[k^{2}\left(\omega V_{12}+\mathrm{i} k \tilde{U}_{2}(\tilde{y})+\frac{k^{2} l}{R e_{2}}\right)+\mathrm{i} k \tilde{U}_{2}^{\prime \prime}(\tilde{y})\right] \tilde{\Psi}_{2}(\tilde{y})=0,
\end{aligned}
$$

where $V_{12}=\bar{U}_{1} / U_{2 \infty}$ is the velocity ratio, subject to the following non-dimensional boundary conditions:

$$
\begin{gathered}
\mathrm{i} \tilde{k} \tilde{\Psi}_{1}( \pm 1)+\left[\tilde{\omega}+\mathrm{i} \tilde{k} \tilde{U}_{1}( \pm 1)\right] \tilde{\eta}_{ \pm}=0 \\
V_{12} \tilde{\Psi}_{1}^{\prime}( \pm 1)-l \tilde{\Psi}_{2}^{\prime}( \pm 1)+\left[V_{12} \tilde{U}_{1}^{\prime}( \pm 1)-\tilde{U}_{2}^{\prime}( \pm 1)\right] \tilde{\eta}_{ \pm}=0 \\
V_{12} \tilde{\Psi}_{1}( \pm 1)-l \tilde{\Psi}_{2}( \pm 1)=0 \\
\tilde{\Psi}_{1}^{\prime \prime}( \pm 1)+\tilde{k}^{2} \tilde{\Psi}_{1}( \pm 1)-M F R \frac{R e_{1}}{R e_{2}} l^{2}\left[\tilde{\Psi}_{2}^{\prime \prime}( \pm 1)+\tilde{k}^{2} \tilde{\Psi}_{2}( \pm 1)\right] \\
+\left[\tilde{U}_{1}^{\prime \prime}( \pm 1)-M F R \frac{R e_{1}}{R e_{2}} l \tilde{U}_{2}^{\prime \prime}( \pm 1)\right] \tilde{\eta}_{ \pm}=0 \\
-\left[\tilde{\omega}+\mathrm{i} \tilde{k} \tilde{U}_{1}( \pm 1)\right] \tilde{\Psi}_{1}^{\prime}( \pm 1)+l M F R\left[\tilde{\omega} V_{12}+\mathrm{i} \tilde{k} \tilde{U}_{2}( \pm 1)\right] \tilde{\Psi}_{2}^{\prime}( \pm 1)-\frac{3 \tilde{k}^{2}}{R e_{1}} \tilde{\Psi}_{1}^{\prime}( \pm 1) \\
+3 \frac{l^{2} M F R}{R e_{2}} \tilde{k}^{2} \tilde{\Psi}_{2}^{\prime}( \pm 1)+\mathrm{i} \tilde{k} \tilde{U}_{1}^{\prime}( \pm 1) \tilde{\Psi}_{1}( \pm 1)-\mathrm{i} \tilde{k} l M F R \tilde{U}_{2}^{\prime}( \pm 1) \tilde{\Psi}_{2}( \pm 1) \\
+\frac{1}{R e_{1}} \tilde{\Psi}_{1}^{\prime \prime \prime}( \pm 1)-\frac{l^{2} M F R}{R e_{2}} \tilde{\Psi}_{2}^{\prime \prime \prime}( \pm 1) \mp \frac{\mathrm{i} \tilde{k}^{3}}{W e} \tilde{\eta}_{ \pm}=0 \\
\tilde{\Psi}_{2}( \pm \infty)=0 \\
\tilde{\Psi}_{2}^{\prime}( \pm \infty)=0
\end{gathered}
$$

In these equations, $R e_{1}, R e_{2}, W e$ and $M F R$ are the Reynolds numbers for the liquid and gas flows, Weber number and momentum flux ratio as defined previously in (3.2) to (3.4).

These equations have been solved employing the Tau-Galerkin projection method, expanding the solution space in Chebyshev polynomials, as illustrated in Orszag (1971) and Gottlieb \& Orszag (1977). The computer code written for this purpose was checked against other published data, in particular, stability of Poiseuille flow (Orszag 1971), temporal stability of the stratified flow of Renardy (1987), and stability results of Teng et al. (1997) for a liquid sheet in confined quiescent air.

\section{Results and discussion}

All the measurements and images collected in the present study confirm some general characteristics of this type of flow that have been described in previous works. In the presence of the high-speed coflowing air streams, the air/water interface interaction results in a Kelvin-Helmholtz-type instability, but modified by the effects of viscosity as will be shown, which quickly cause the sheet breakup. As explained for example in Mansour \& Chigier (1990), this fast atomization occurs for air/water velocity ratios for which sinusoidal waves are dominant over dilatational ones. This regime is defined by Mansour \& Chigier (1991) as zone B, where the breakup mode 

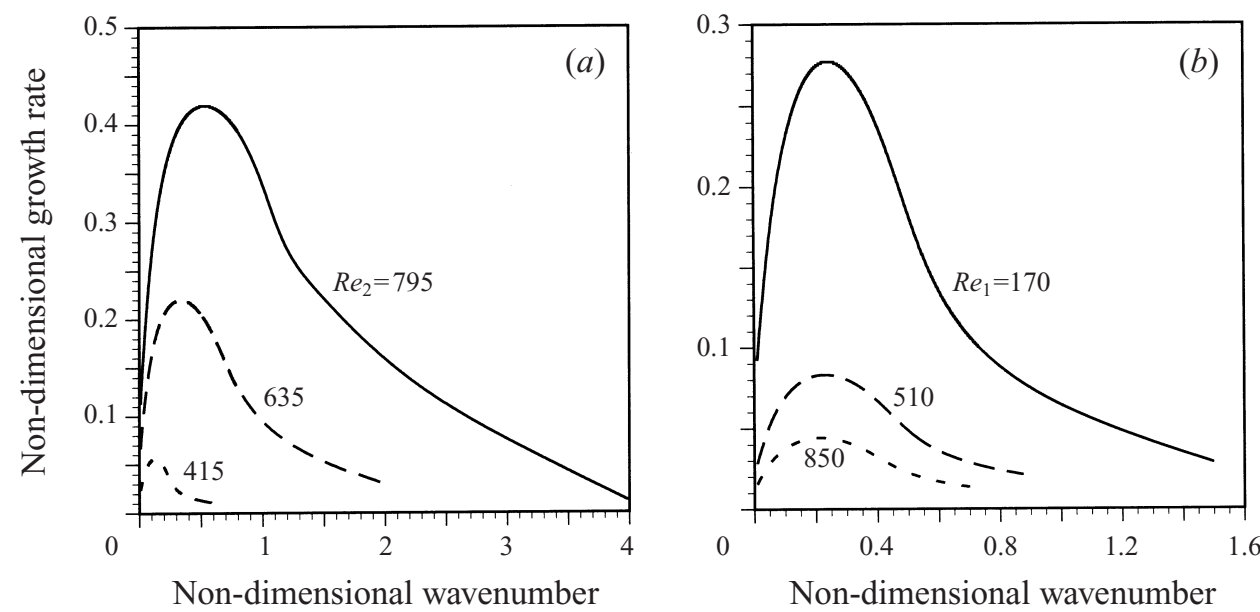

FIGURE 6. Non-dimensional growth rates as a function of the non-dimensional wavenumbers: (a) for a fixed $R e_{1}=170$ (water velocity $\bar{U}_{1}=1 \mathrm{~m} \mathrm{~s}^{-1}$ ) and varying $R e_{2}\left(U_{2 \infty}=15,35,55 \mathrm{~m} \mathrm{~s}^{-1}\right)$; (b) for a fixed $R e_{2}=535$ (air velocity $U_{2 \infty}=25 \mathrm{~m} \mathrm{~s}^{-1}$ ) and varying $R e_{1}\left(\bar{U}_{1}=1,3,5 \mathrm{~m} \mathrm{~s}^{-1}\right.$ ).

exhibits streamwise ligaments (Stapper \& Samuelsen 1990). For lower air/water velocity ratios there is a zone $\mathrm{C}$ characterized by the presence of dilatational waves that prevent the dominant growth of the sinusoidal ones, which results in a poor atomization, with reduced spray angles and droplets of larger diameters. Mansour \& Chigier (1991) define also a zone A, for low water velocities, where the sheet breakup in longitudinal filaments occurs at the nozzle lip and no intact sheet length is visible. Examples of the sheet appearance in each one of these zones can be found in Mansour \& Chigier (1991) or Lozano et al. (1996).

The best atomization characteristics occur in zone B, with a dominant sinusoidal oscillation of the liquid sheet, rapid amplitude growth, large spray angle and small droplet sizes. In this regime, linear perturbation growth only takes place for distances of the order of one or two half-wavelengths. Recall that a linear regime indicates that the problem can be satisfactorily described by the linearized Navier-Stokes equations, and that the predicted growth of the propagating longitudinal waves is, under these conditions, exponential without distortion in the wave shape. For longer distances, the growth regime is nonlinear and wave profiles assume a zigzag shape in what Mansour \& Chigier (1990) describe as 'half waves torn off the disturbed sheet'. It is at this moment that atomization begins.

The numerical predictions are intended to reproduce the initial stage of the instabilities, and when they depart from the linear regime the present numerical analysis is obviously inadequate. Furthermore, according to the experimental evidence, only sinusoidal oscillations such as those observed in zone B have been reproduced. This is the reason why dilatational waves have not been considered.

In this work, a temporal stability analysis was performed. For a given set of variable values, a complex temporal frequency value $\omega$ was obtained for each real wavenumber $k$. Temporal growth occurs for positive real part $\omega_{r}>0$. The imaginary part of the temporal frequency $\omega_{i}$ corresponds to the wave oscillation frequency. To illustrate this procedure, figure 6 presents examples of the plots obtained when solving the dispersion relation. In figure $6(a)$ the air velocity was varied while keeping the water velocity constant and equal to $1 \mathrm{~m} \mathrm{~s}^{-1}$. On the other hand, in figure $6(b)$ the air velocity was fixed to $25 \mathrm{~m} \mathrm{~s}^{-1}$, and the water velocity was varied. It can be observed 


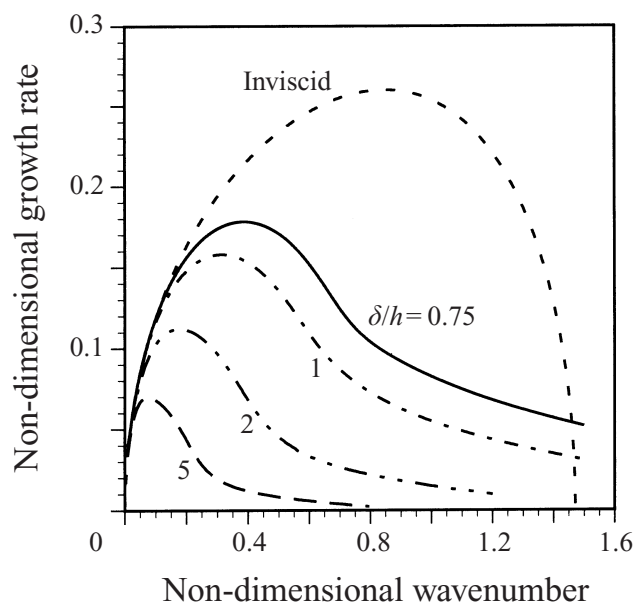

FIGURE 7. Non-dimensional growth rate $v$ s. non-dimensional wavenumber for fixed $R e_{1}=340$ $\left(\bar{U}_{1}=2 \mathrm{~m} \mathrm{~s}^{-1}\right)$ and $R e_{2}=535\left(U_{2 \infty}=25 \mathrm{~m} \mathrm{~s}^{-1}\right)$, and varying boundary layer thickness $\delta$, expressed as the ratio $\delta / h$ where $h$ is the sheet half-thickness.

that all the curves have a range for which the growth rate is positive, i.e. for which unstable waves can propagate, and all of them present a maximum for a determinate wavenumber, corresponding to the most unstable mode.

To obtain predictions that could be compared to the experimental measurements, the equations were solved for the same ranges of gas and liquid exit velocity as those in the experiments. As fluid parameters (density, viscosity and surface tension) those of air and water were selected. The liquid sheet thickness was fixed in all the cases to the experimental one, $0.35 \mathrm{~mm}$. Finally, the air boundary layer thickness was varied in different runs because analysing its influence on the results was one of the main objectives of the present research. No accurate measurements of this parameter were experimentally obtained, but from particle image velocimetry (PIV) images it was determined to be less than $0.4 \mathrm{~mm}$ at the nozzle exit for an air velocity of $25 \mathrm{~m} \mathrm{~s}^{-1}$. This thickness was also estimated assuming a laminar boundary layer Blasius profile.

Although the equations were solved in non-dimensional form, to ease the comparison with the experiments, most of the results are presented in physical units. It was also considered convenient to present the measurements in dimensional plots, both to facilitate comparisons with other papers and to relate them more easily to the actual experimental runs, where normally variables such as velocity rather than non-dimensional groups are controlled.

\subsection{Effects of air boundary layer thickness in the linear instability analysis}

For a fixed water velocity of $2 \mathrm{~m} \mathrm{~s}^{-1}$ and an air exit velocity of $25 \mathrm{~m} \mathrm{~s}^{-1}$, figure 7 shows the effect of varying the air boundary layer thickness. It can be observed that increasing the thickness causes a decrease in the growth rate, as well as in the wavenumber for which the maximum growth is reached. This is also accompanied by a reduction in the oscillation frequency. The dashed line corresponds to the analytical solution of the inviscid problem, i.e. considering constant basic velocity profiles both for water and air, as well as inviscid perturbations. Observe that, in this case, all the viscous terms and the derivatives of the steady state velocities vanish, and the dispersion relation reduces to a second-order algebraic equation. In this limit, the boundary layer thickness is zero. Each curve in figure 8 has been obtained by plotting 


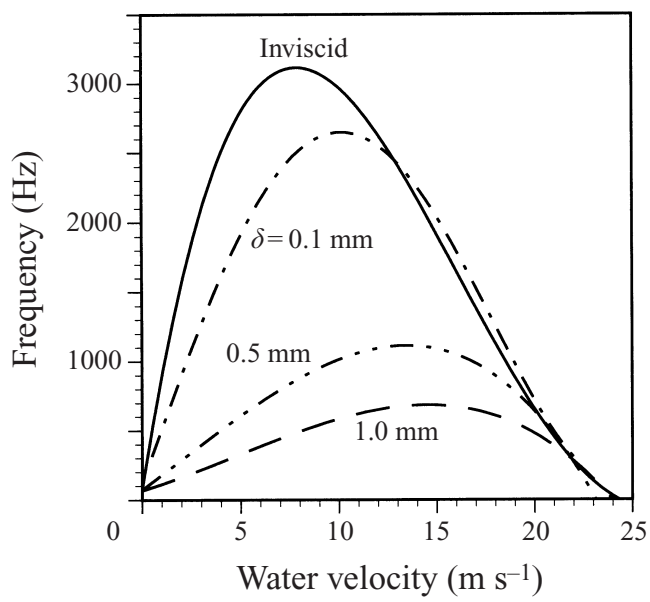

FIGURE 8. Maximum oscillation frequencies for fixed air velocity $U_{2 \infty}=25 \mathrm{~m} \mathrm{~s}^{-1}$, and varying water velocities, for different air boundary layer thicknesses.

the frequencies of maximum growth for different water velocities while keeping the air velocity fixed at a value of $25 \mathrm{~m} \mathrm{~s}^{-1}$. It is interesting to observe how increasing the boundary layer thickness reduces the value of the oscillation frequencies, although slightly increasing the water velocity value for which the oscillation frequency displays a maximum. A thicker air boundary layer acts to damp the oscillation of the liquid sheet, simultaneously reducing the maximum growth rate and the frequency for which this maximum is attained, and increasing the wavelength of the propagating perturbation.

The first and very important conclusion is that the boundary layer thickness has a strong influence on the oscillation frequency values, and consequently, it is a parameter that cannot be ignored if quantitative predictions are to be attempted. This implies that quantitative results obtained from inviscid approximations have to be critically questioned.

\subsection{Oscillation frequency results}

Figure 9 summarizes the frequency measurements obtained in this work. They are plotted as a function of water velocity for the different air velocity values. This choice of variables was selected so that the plots can be more easily compared with previously reported measurements. The present results are in very good agreement with those given in Mansour \& Chigier (1991) and Lozano et al. (1996). The dashed line in figure 9 roughly delimitates zones B and C (cf. figure 9 in Mansour \& Chigier). Zone A would appear at the left of zone B in the figure but has not been measured in these experiments. For the air and water velocities considered in this study, the oscillation frequency increases linearly for increasing air exit velocities, showing a much weaker dependence on water velocity. It can be observed, however, that most of the frequency curves as a function of water velocity have a maximum value in zone B.

In figure 10, the same frequency measurements are plotted as a function of air velocity. The functional dependence is approximately linear. The straight line in the figure fits the frequency values for a constant water velocity $\bar{U}_{1}=3.15 \mathrm{~m} \mathrm{~s}^{-1}$. Similar lines can be obtained by fitting the frequency measurements for the rest of the water velocities. Performing these fits, it can be seen that the resulting straight lines do not 


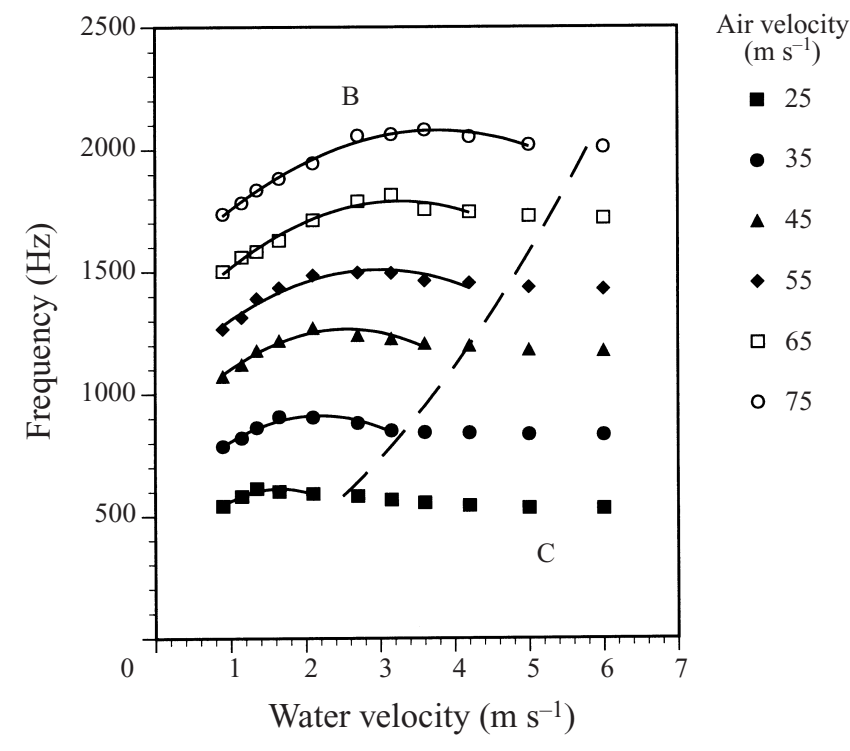

FIGURE 9. Measurements of the liquid sheet oscillation frequency as a function of water exit velocity, for different air velocities. The dashed line indicates the transition from zone B to C, as defined in Mansour \& Chigier (1991).

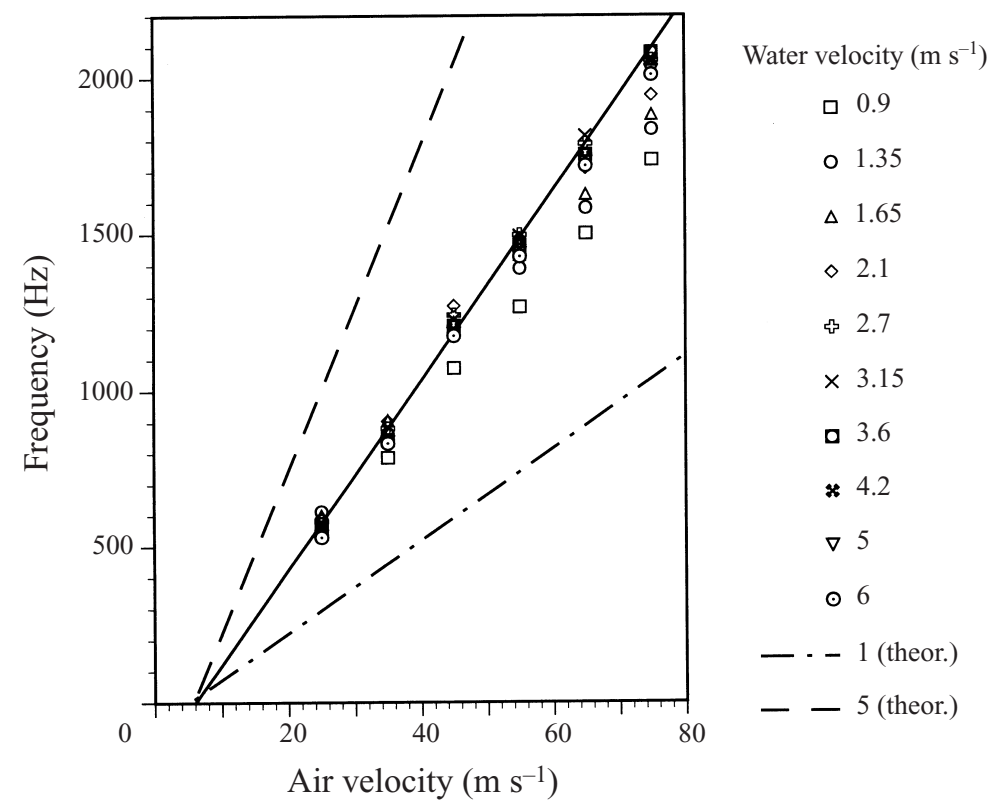

FIGURE 10. Measurements of the liquid sheet oscillation frequency $v$ s. air velocity. The straight solid line is fitted to the frequency values corresponding to $\bar{U}_{1}=3.15 \mathrm{~m} \mathrm{~s}^{-1}$. Dashed lines are numerical results for $\bar{U}_{1}=1$ and $5 \mathrm{~m} \mathrm{~s}^{-1}$.

pass through the origin but intersect the abscissa at non-zero values. This fact can be explained by associating these values with a minimum air speed, $U_{\min }$, that would be necessary to sustain a sinusoidal oscillation for a given water velocity. This limit will never be reached in real experiments because as the air/water velocity ratio decreases, dilatational waves are also present, and the oscillation behaviour corresponds to zone 


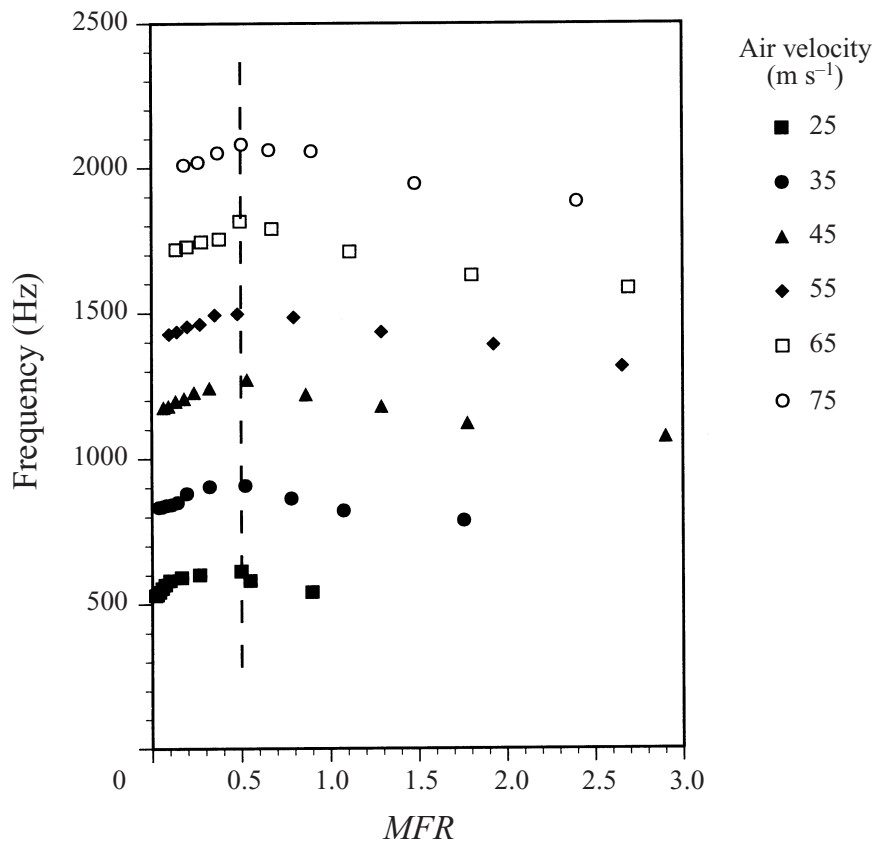

FIGURE 11. Sheet oscillation frequency plotted against momentum flux ratio $(M F R)$. The straight line correspond to the maxima in the curves of figure $4(M F R=0.5)$.

$\mathrm{C}$ described above with no dominant sinusoidal mode. From the measurements, it was observed that $U_{\min }$ follows a quadratic dependence with water velocity, and the same happens to the slope of the straight lines.

In figure 11 the measurements presented in figure 9 are displayed by plotting the frequency values as a function of the momentum flux ratio $(M F R)$ as defined in $\S 3$. It can be observed that for all the air velocity values, the frequency maxima on varying the water velocity correspond to a fixed $M F R$ value of 0.5 . The dashed curve separating zones $\mathrm{B}$ and $\mathrm{C}$ in figure 9 corresponds to $M F R$ values ranging from 0.1 to 0.2 for increasing air velocities up to $75 \mathrm{~m} \mathrm{~s}^{-1}$. This line indicates the transition to a dominant sinusoidal oscillation mode, and it is interesting to note that it occurs for a much higher momentum flux (or, equivalently, dynamic pressure) in the water than in the air. These alignments corroborate the relevance of the MFR as an appropriate non-dimensional parameter to characterize the problem. All the curves can be approximately collapsed if the frequency axis is non-dimensionalized in the form

$$
f^{*}=\frac{f d}{U_{2 \infty}-U_{\min }},
$$

where $d=2 h$ is the sheet thickness and $U_{\min }$ has been introduced according to the experimental evidence, as explained in the paragraph above. A single $U_{\min }$ value, the one corresponding to the line joining the maximum oscillation frequencies, has been selected. The final result is depicted in figure 12, and although the collapse is not complete (recall that $U_{\min }$ depends on water velocity), the result is quite satisfactory.

A question has recently been raised about the possible mechanism that could trigger the initial longitudinal perturbation that is later amplified, causing the sheet breakup. A first explanation could relate it to the air vortex shedding from the 


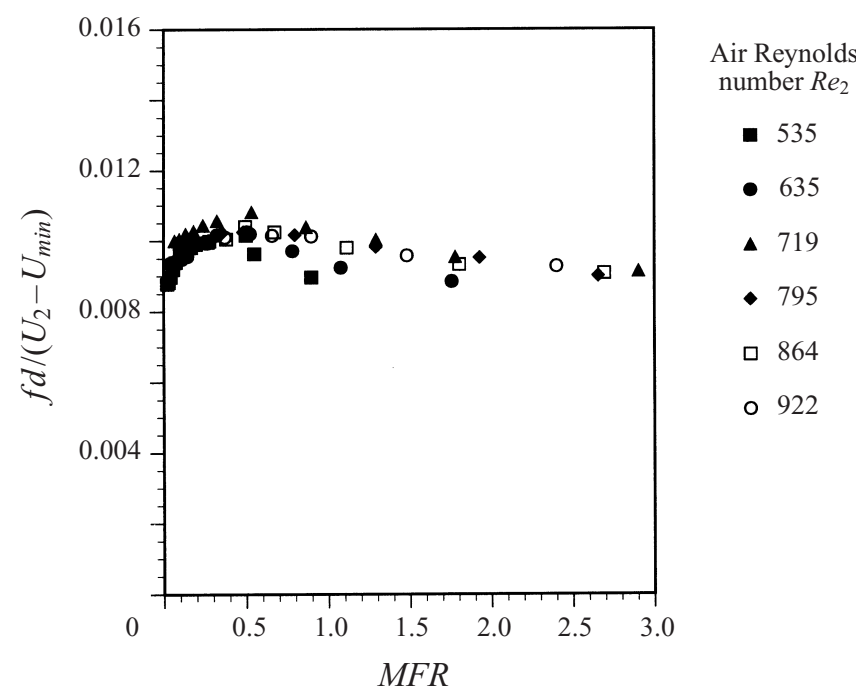

FigURE 12. Same plot as figure 11 with the ordinate axis non-dimensionalized by the sheet thickness and the air velocity (see text). $R e_{2}$ values correspond to the air velocities in figure 11 . All the curves collapse into a single one.

nozzle lip, recalling the strong dependence of the oscillation frequency on the air velocity. A comparison with analogous situations in similar flows could be made. The unforced vortex shedding past a solid cylinder i.e. the typical Kármán vortex street, occurs with a Strouhal number of 0.21 defined with the free-stream velocity and a characteristic body length, in this case, the cylinder diameter. Translating the analogy to the present case, a pertinent length scale should be the sheet thickness plus the thickness of the nozzle lips. Substituting the frequency measurements, air velocities and length scale values for these experiments, a Strouhal number of 0.025 is obtained, demonstrating that this problem responds to different underlying physical mechanisms. Another situation that could be similar to this problem would be the vortex shedding from a backward-facing step. Experiments reported in the literature indicate a Strouhal number of 0.1 for this flow (Eaton \& Johnston 1980). In this analogy, it seems that the appropriate length scale should be the nozzle lip, which for the present experiments yields a Strouhal number of 0.01 . However, it is obvious that the possibility of sheet displacement makes this case significantly different from shedding over a fixed solid wall. The onset of the sheet oscillation with the water surface that is moving sinusoidally modifies the pressure and velocity fields in the neighbourhood of the air vortices to be detached. The air, in turn, must displace the water sheet with its large inertia. These changes could explain why a lower oscillation frequency is observed.

The fact that the liquid mass is influential in the sheet flapping is confirmed by numerous measurements indicating that the oscillation frequency decreases with increasing sheet thickness. If the sheet thickness is substituted in the expression for the Strouhal number, similar values are obtained from different experiments: 0.01 in the present case, 0.0067 from Mansour \& Chigier (1991) and 0.007 from Lozano et al. (1996). This agreement reinforces the assumption that the sheet thickness is the appropriate length scale characterizing the oscillation. The initial displacement of the liquid sheet, together with the location of the triple contact line among the air, liquid and solid nozzle surface, which could be moving at the lip edge, seem to act jointly 


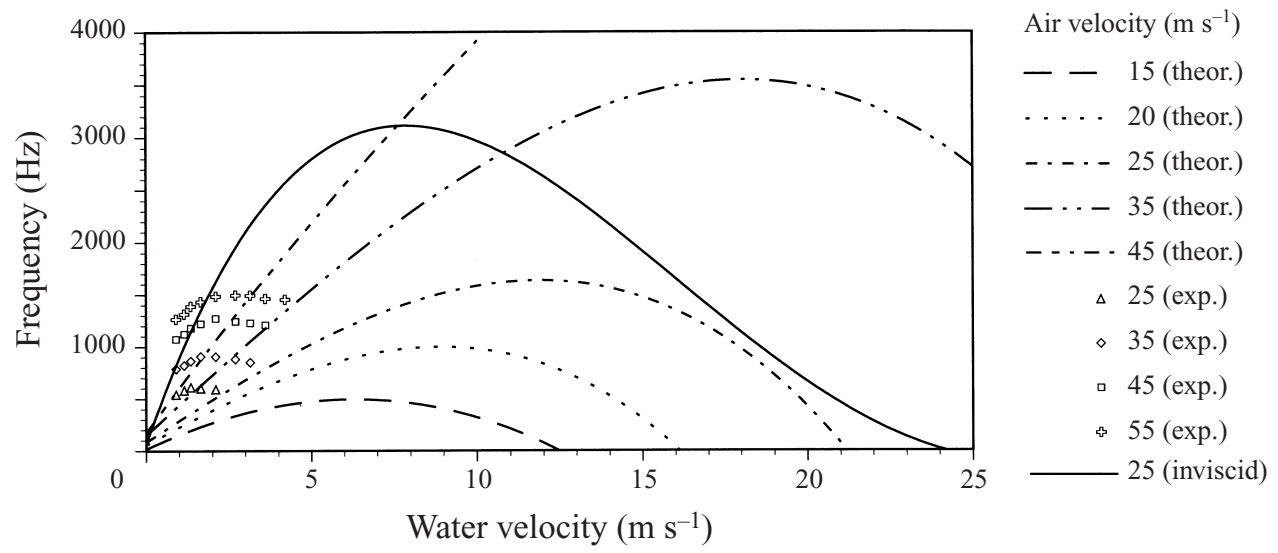

FIGURE 13. Numerical predictions of the oscillation frequency as a function of water exit velocity, for different air velocities. Some experimental curves from figure 9 are included for comparison. A curve obtained from the inviscid linear instability analysis is also included.

to minimize the effect of the nozzle lip thickness on the shedding of the vortices that could trigger the sheet oscillation. The small discrepancy among the resulting Strouhal numbers $(0.01,0.007,0.0067)$ might be due to the fact that the present experiments are the only ones where air and water exit velocities were parallel, whereas in Mansour $\&$ Chigier and Lozano et al. air was impinging onto the water sheet at an angle. This result points to the interesting fact that to atomize it might be more efficient to inject parallel air and liquid streams rather than flowing the air at an angle with respect to the sheet. Unfortunately, with the present nozzle assembly the air exit angle cannot be varied.

The frequency values predicted from the linear instability analysis for a given set of air and water velocities, to be compared with the experimental measurements, correspond to those for which the growth rate is a maximum. It is assumed that being the ones with a faster growth, they should be dominant, and consequently, the ones experimentally observed.

Frequency values were calculated for different air and water exit velocities. The air boundary layer width was varied with the air velocity according to a $U_{2 \infty}^{-1 / 2}$ law. All the resulting frequencies are plotted in figure 13 and are to be compared with the experimental measurements in figure 9. It is observed that for each air velocity, the frequency has a maximum for a given water velocity, in qualitative agreement with the experimental behaviour. This trend is also reproduced if the linear analysis is performed for inviscid fluids. However, the values predicted when viscous effects are included are closer to the measured ones than those obtained in the inviscid limit. However, although results improve when viscosity is included, and the functional dependence of the frequency on air and water velocities is properly captured, the quantitative agreement is not satisfactory.

It is interesting to observe that while the experimental frequency curves in zone B are accurately approximated by quadratic functions, the linear instability results are better fitted to third-order polynomials.

The numerical frequency predictions can also be plotted as a function of the air velocity (figure 14). The most remarkable characteristic of this plot is that the linear dependence of the oscillation frequency on air velocity observed in the measurements is computationally reproduced. This is especially interesting because if viscosity is 


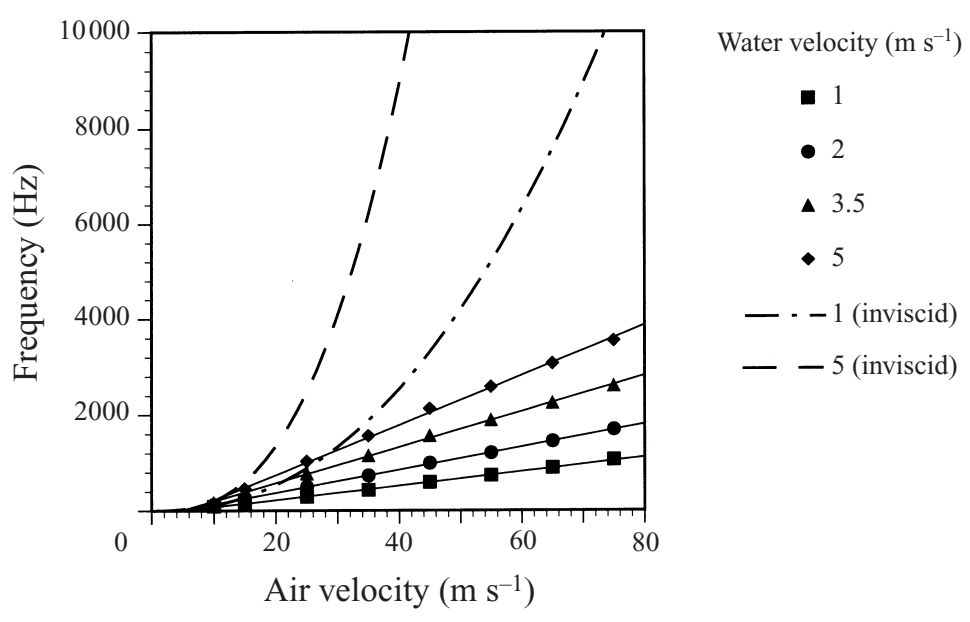

FIGURE 14. Numerical predictions of the liquid sheet oscillation frequency vs. air velocity. Two curves obtained from the inviscid linear instability analysis are also included for comparison.

ignored, the predicted dependence becomes quadratic as depicted by the dashed line in the figure. A pure inviscid Kelvin-Helmholtz instability mechanism only driven by pressure variations is unable to explain the experimentally observed oscillations. These results reveal for the first time that the air viscosity has a significant role in fixing the sheet oscillation frequency even in the linear regime, and questions once again the validity of some inviscid analysis.

A comparison with the experimental data is also displayed in figure 10. It is evident that although there will be agreement for a particular water velocity (around $2 \mathrm{~m} \mathrm{~s}^{-1}$ ), as this velocity is increased or decreased predicted values depart from the measured ones.

\subsection{Droplet size distributions}

Droplet size distributions were obtained by laser diffractometry. From them, the Sauter mean diameter, $S M D$, defined in (2.1) was calculated. Results are presented in figure $15(a)$. As in figure 9, the dashed line defines the transition between oscillation regimes $\mathrm{B}$ and $\mathrm{C}$. It is evident that the mean diameter decreases with increasing air velocity. For a fixed air velocity, and varying water velocities, the mean diameter appears to present a minimum roughly coincident with the frequency maximum (compare Figures 9 and 15a). This minimum is more pronounced for low air speeds. When crossing from zone $\mathrm{B}$ to $\mathrm{C}$, i.e. decreasing the momentum flux ratio $M F R$ below $0.2-0.1$, the mean diameter increases, due to the different sheet oscillation characteristics. On the other hand, an increase in MFR does not necessarily cause a decrease in droplet diameter from primary atomization. It has to be noted, however, that when measuring at larger downstream distances $(20$ or $30 \mathrm{~cm})$ for a fixed air velocity the diameter always tends to decrease for increasing $M F R$, due to secondary breakup.

The SMD dependence on air velocity is more clearly seen in figure $15(b)$. The measured points in the figure have been adjusted to a $1 / U_{2 \infty}$ function. Note that the mean diameter dependence on air and water velocities is similar to that of the streamwise filament spacing. This can be observed by comparing figures $15(a)$ and 15(b) with figures 10 and 11 in Lozano et al. (1996). The similar behaviour displayed 

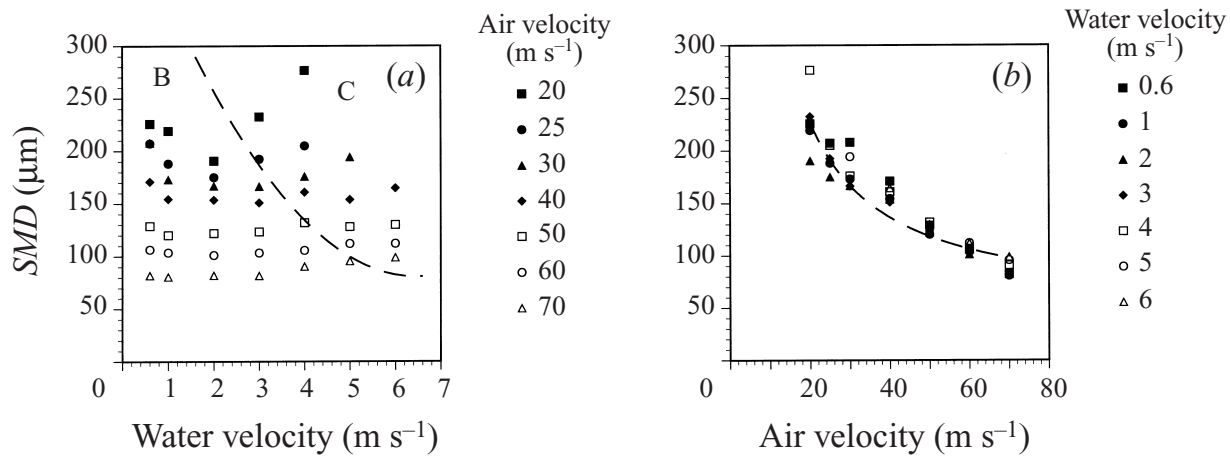

FIGURE 15. (a) Sauter mean diameter (SMD) measurements as a function of water exit velocity, for different air velocities. The dashed line indicates the transition from zone B to C, as in figure 9 . (b) $S M D$ as a function of air velocity. The dashed line is a fit to a $1 / U_{2 \infty}$ function.

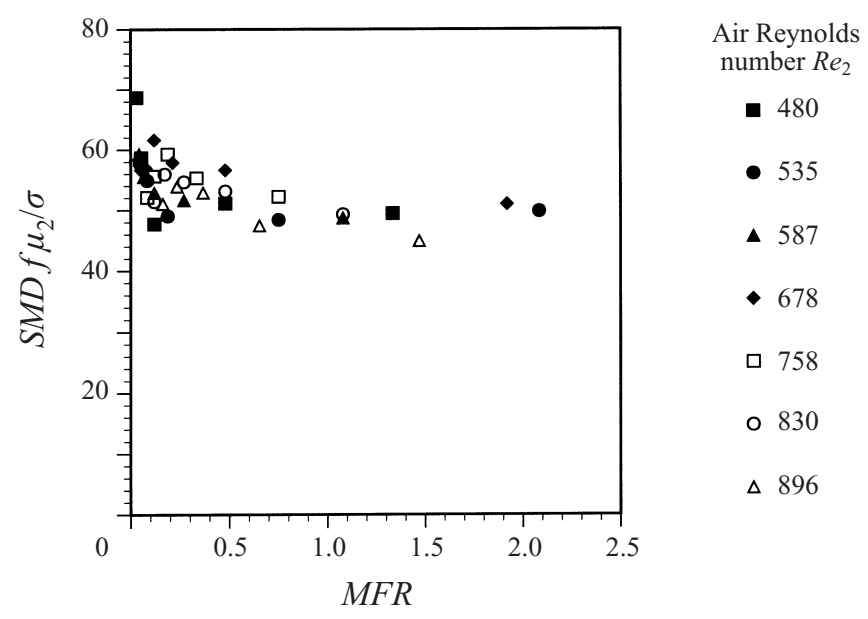

FIGURE 16. Non-dimensional plot of $\left(S M D f \mu_{2}\right) / \sigma$ as a function of $M F R$. The air Reynolds numbers $\left(R e_{2}\right)$ correspond to the air velocities in figure 15 .

by both parameters indicates that a relation exists between droplet diameter and filament spacing.

The inverse correlation between $S M D$ and oscillation frequency suggests it might be convenient to attempt to collapse all the curves in figure $15(a)$ in a similar way to that followed to obtain figure 12. MFR has been selected as the parameter on the abscissa, again capturing the dependence on air and water velocity. To collapse the vertical axis, a non-dimensional parameter was defined, including physical properties that are known to be influential in the droplet diameter resulting from the atomization processes. The selected parameter is:

$$
\frac{S M D f \mu_{2}}{\sigma}
$$

where $f$ is the sheet oscillation frequency, $\mu_{2}$ is air viscosity and $\sigma$ is the surface tension coefficient. The final result, which can be considered to be satisfactory, is depicted in figure 16. 
(a)

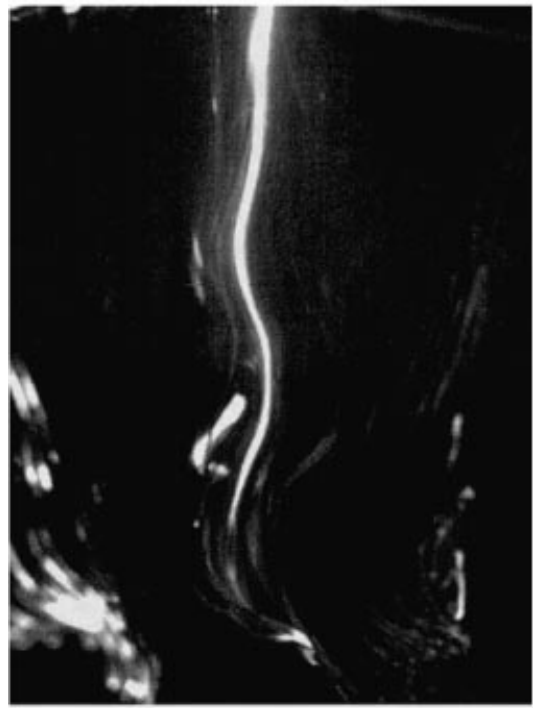

(c)

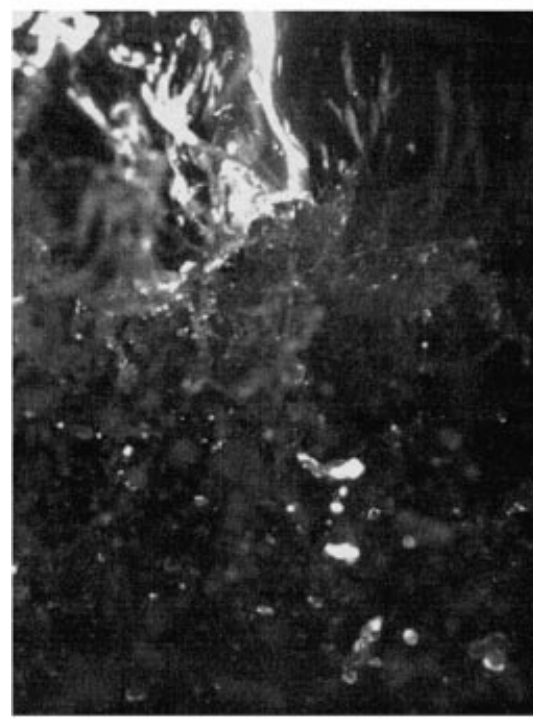

(b)

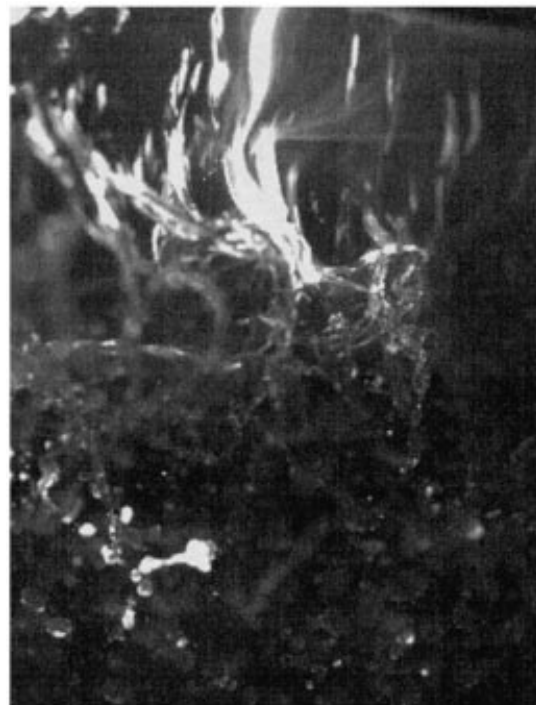

(d)

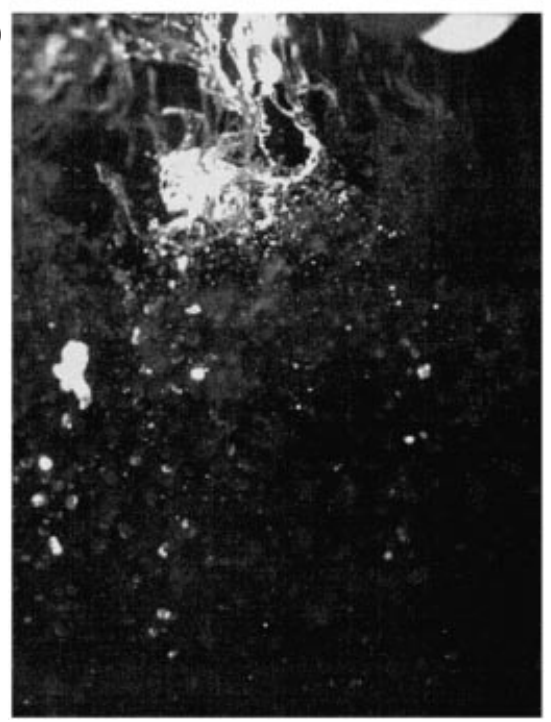

FIGURE 17. Luminous trace indicating the intersection between the oscillating water sheet and the illumination laser plane. Field of view is $26 \times 35 \mathrm{~mm}$. The nozzle was located at the top of the image. Note that the sheet is viewed with a $25^{\circ}$ angle. Water exit velocity: $1.2 \mathrm{~m} \mathrm{~s}^{-1}$. Air velocities: (a) $15 \mathrm{~m} \mathrm{~s}^{-1}$, (b) $25 \mathrm{~m} \mathrm{~s}^{-1}$, (c) $30 \mathrm{~m} \mathrm{~s}^{-1}$, (d) $40 \mathrm{~m} \mathrm{~s}^{-1}$.

\subsection{Wavelength results}

Examples of the instantaneous images acquired to study the oscillation wavelength are depicted in figures 17 and 18 . The field of view in these images is $26 \times 35 \mathrm{~mm}$ extending downstream from the nozzle lip, with a resolution of $90 \mu \mathrm{m} /$ pixel. Some ambiguity exists in defining a perturbation wavelength because as the wave propagates with the accelerating water sheet, its velocity increases while it moves downstream. As the frequency has been experimentally determined to remain constant with downstream distance (cf. Mansour \& Chigier), the wavelength must grow according to the relation $f \lambda=c$, where $f$ is the oscillation frequency, $\lambda$ is the wavelength and $c$ is the phase velocity. This type of phenomenon is perfectly illustrated in figure 1(c) of De Kee 
(a)

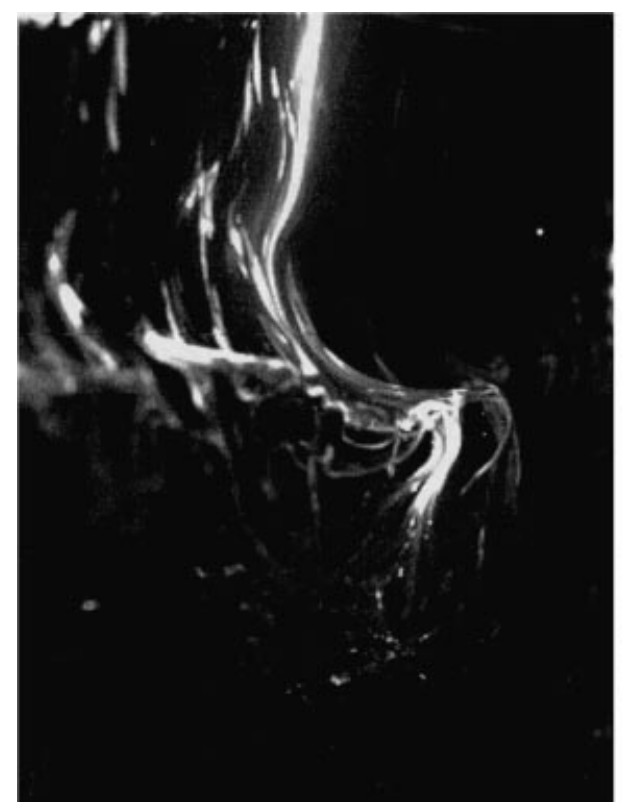

(b)

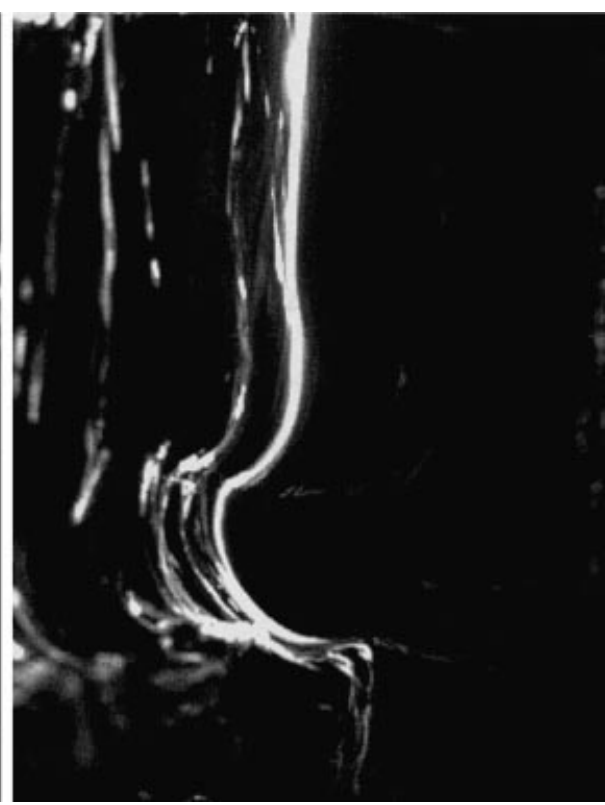

FIGURE 18. Similar images as in figure 18 for air velocity of $25 \mathrm{~m} \mathrm{~s}^{-1}$ and water velocities of (a) $2.4 \mathrm{~m} \mathrm{~s}^{-1}$ and (b) $3.0 \mathrm{~m} \mathrm{~s}^{-1}$.

\& Wissburn (1998) for a non-Newtonian fluid. Despite this, the images obtained are revealing, because comparisons can be made among the waves resulting for different air and water velocities, and for a variety of MFR values.

Figure 17 shows instantaneous images for a fixed water velocity of $1.2 \mathrm{~m} \mathrm{~s}^{-1}$ and increasing air speeds. From left to right and top to bottom, the air velocities are 15, 25, 30 and $40 \mathrm{~m} \mathrm{~s}^{-1}$ (MFR of $0.18,0.5,0.73$ and 1.29). The breakup length clearly decreases for increasing air exit velocity. If the air velocity is maintained at a fixed value, it can be observed that the breakup distance increases with water velocity. Figure 18 displays an example for air velocity of $25 \mathrm{~m} \mathrm{~s}^{-1}$ and water velocities of $2.4 \mathrm{~m} \mathrm{~s}^{-1}(a)$ and $3.0 \mathrm{~m} \mathrm{~s}^{-1}(b)$.

Extending these conclusions to the wavelength behaviour proves somewhat harder. This is aggravated by the fact that the sinusoidal waves become highly distorted prior to breakup, which takes place after only one or two wavelengths. With this in mind, and accepting errors as large as $25 \%$ in the wavelength measurements, the general behaviour is summarized in figure 19 , where the wavelength in $\mathrm{mm}$ is plotted $v \mathrm{~s}$. water velocity, for different air velocities. A sudden increase in wavelength for increasing water velocity is apparent when the oscillation regime transitions from zone B to C (limit represented as a dashed line in the figure).

With the present numerical code, wavelengths for which the wave growth rate is maximum can be directly obtained by inverting the critical wavenumbers. Results are also included in figure 19, represented with hollow symbols. The predictions slightly underestimate the experimental measurements for low air velocities. However, as the air velocity increases, numerical and measured results are quite close. The code is, in any event, unable to capture the $\mathrm{B}$ to $\mathrm{C}$ regime transition. Should the wavelengths be calculated using the inviscid instability analysis the values would be significantly 


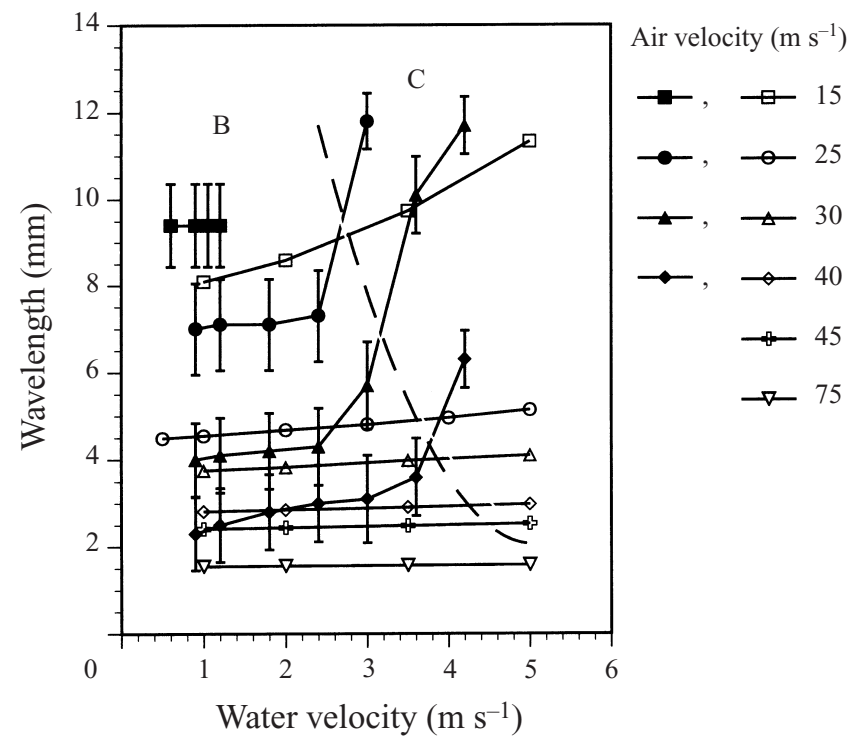

FIGURE 19. Measured (solid symbols) and calculated (hollow symbols) wavelength in $\mathrm{mm}$ as a function of water velocity, for different air velocities. The dotted line at the right indicates the transition between zones $\mathrm{B}$ and $\mathrm{C}$ for the measured values.

(a)

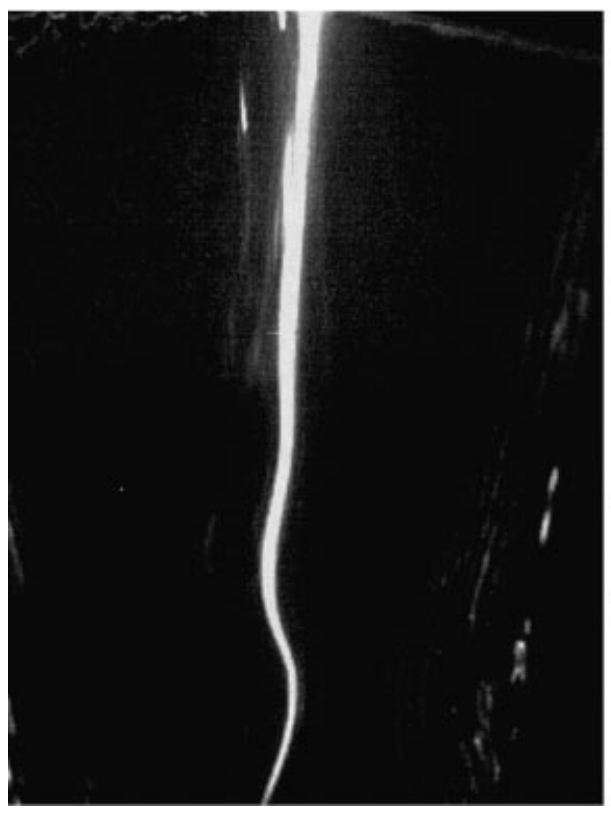

(b)

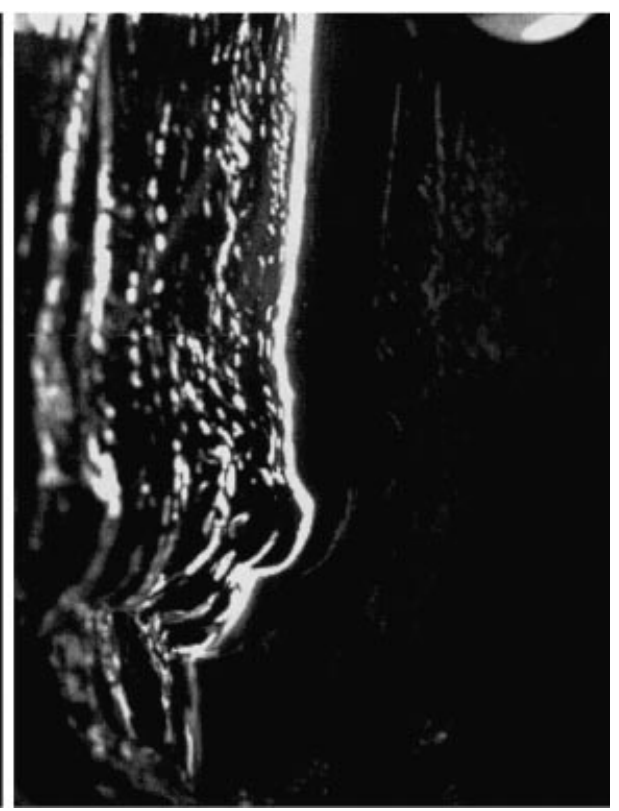

FIGURE 20. Longitudinal sections of the sheet for the same MFR value (0.09) and different velocities: (a) $\bar{U}_{1}=1.75 \mathrm{~m} \mathrm{~s}^{-1}, U_{2 \infty}=15 \mathrm{~m} \mathrm{~s}^{-1} ;(b) \bar{U}_{1}=4.5 \mathrm{~m} \mathrm{~s}^{-1}, U_{2 \infty}=40 \mathrm{~m} \mathrm{~s}^{-1}$.

lower than the results obtained when viscosity is included, and thus the agreement with the measurements would be poorer.

Figure 20 presents two images with very similar MFR (0.09) but different velocities $\left(U_{1}=1.75\right.$ and $4.5 \mathrm{~m} \mathrm{~s}^{-1}, U_{2 \infty}=15$ and $\left.40 \mathrm{~m} \mathrm{~s}^{-1}\right)$. For this particular MFR value, the liquid sheet in both images seems to be oscillating with a similar longitudinal 

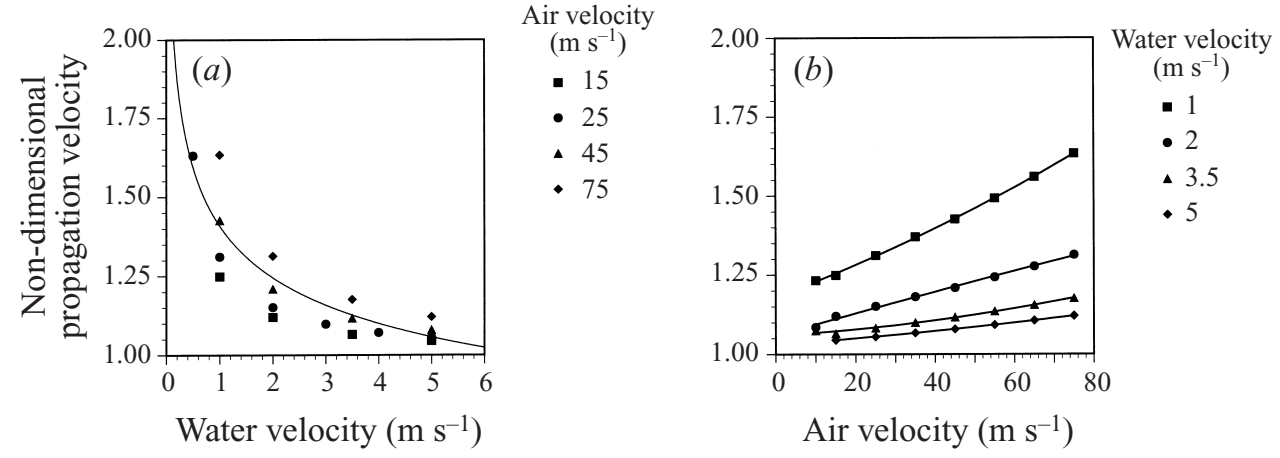

FIGURE 21. Predicted non-dimensional wave propagation velocity as a function of $(a)$ water velocity for different air speeds (the solid line is a fit to the calculated velocities), and $(b)$ air velocity for different water speeds.

wave. However, while in $(a)$ no transverse structure can be discerned, $(b)$ shows a series of striations, indicating the presence of transverse waves. Transverse waves appear to be the most probable cause of the formation of longitudinal filaments (Lozano, García-Olivares \& Dopazo 1998). From this image, it might be inferred that their frequency should mainly depend on the water velocity. On the contrary, from the present experiment observations, varying the air and water conditions it can be concluded that it is the air velocity that ultimately determines the frequency of the transverse wave of maximum growth, much in the same way as with the longitudinal waves. Filament spacing is principally fixed by the air velocity.

\subsection{Wave propagation speed}

To experimentally determine the wave propagation velocity at the nozzle exit is somewhat difficult because it varies with the downstream distance. Furthermore, application of a time-of-flight measurement between two fixed points is not straightforward due to the small wavelength values (less that $1 \mathrm{~cm}$ ). If, as an approximation, it is assumed that the interface velocity is the mean between the air and water speeds, following the indications of many papers available in the literature, the results obtained, for example, when calculating wavelengths from the frequency measurements are much larger than the measured ones. A better agreement between the range of measured and predicted values is obtained if $c$ is assumed to be the water velocity, which for simplicity can be approximated to its exit velocity.

Wave propagation speeds have also been calculated numerically. For any set of flow conditions, the present linear analysis provides for each wavenumber $k$ the corresponding complex temporal frequency $\omega$, formed by the imaginary part $\omega_{i}$, that gives the oscillation frequency, and real part $\omega_{r}$ that corresponds to the growth rate. With this information, it is immediately possible to calculate the wave propagation speed $c$ according to the relation $c=\omega_{i} / k$. This type of result is usually absent in most papers on related subjects, especially those considering water exiting into a quiescent atmosphere, because for ease of calculation, it is customary to assume that the propagation speed is identical to that of the water. Here, it is possible to test this common assumption.

Figure 21(a) shows the calculated variation of the propagation speed divided by the water exit velocity $c_{i}=c / \bar{U}_{1}$ as a function of the water velocity for different air speeds. Figure 21(b) displays the variation of the same non-dimensional propagation 


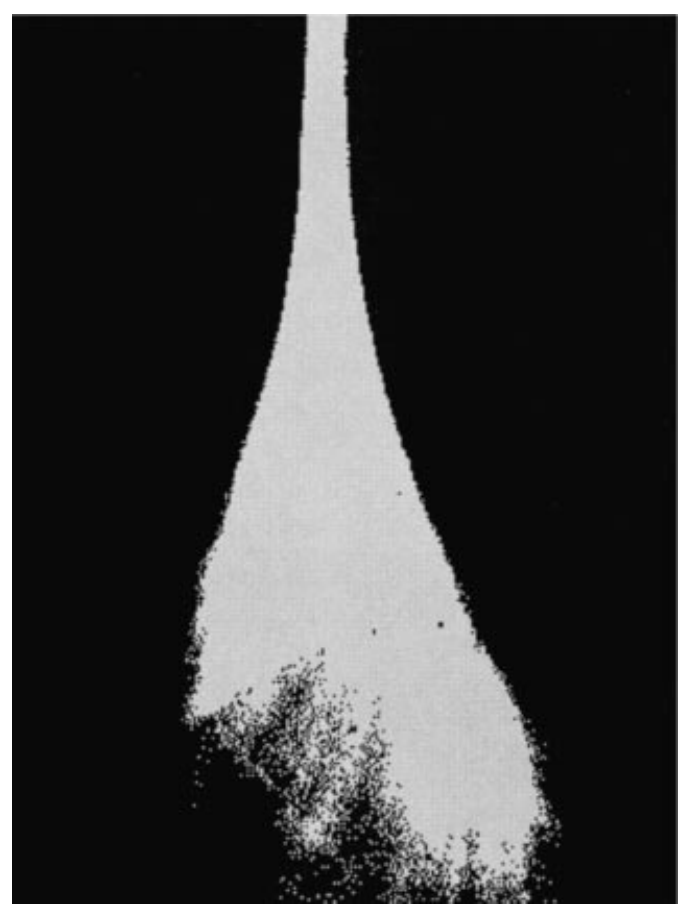

FIGURE 22. Average image of 1000 single-shot images of the longitudinal section of the sheet, for air and water velocities of $15 \mathrm{~m} \mathrm{~s}^{-1}$ and $0.6 \mathrm{~m} \mathrm{~s}^{-1}$ respectively.

speed as a function of air velocity for several water velocity values. In the present calculations, the resulting values range from 1 to 1.6 , thus the actual propagation speed exceeds the water velocity. It is observed that for high air/water velocity ratios, the air quickly accelerates the sheet, and the resulting dimensionless propagation speed $c_{i}$ is higher than 1 . However, as the water velocity increases, the air accelerating effect is weakened, and the value of $c_{i}$ approaches 1 .

\subsection{Oscillation-amplitude growth rate}

As explained in $\S 2$, oscillation-amplitude growth rates have been obtained from averaged flow images, fitting the interface location to an exponential curve of the form $\eta=\eta_{0} \exp \left(k_{r} x\right)$, where $k_{r}$ is the growth factor of a sinusoidal wave described by $\eta=\eta_{0} \exp \left(\left(k_{r}+\mathrm{i} k_{i}\right) x+w t\right)$. To determine the interface location the images have been thresholded and binarized. An example of these images, already processed, is displayed in figure 22, where air and water velocities are 15 and $0.6 \mathrm{~m} \mathrm{~s}^{-1}$, respectively. Figure 23 is an example of how the exponential function is adjusted to the interface profile. As the vertical axis is shown in logarithmic coordinates, the fitted function becomes a straight line. The line adjusts accurately to the initial section of the interface. The last points are slightly displaced, indicating that for these downstream distances, the wave is no longer in the linear regime.

This procedure confirms that, effectively, in the initial instability development process, there is a region of exponential growth. Although this fact has always been assumed, experimental confirmations had not been published before. In fact, these results substantiate the possibility of trying to describe the instability growth using a linear analysis.

From the instability analysis, the calculated growth rate is the real part $\omega_{r}$ of the 


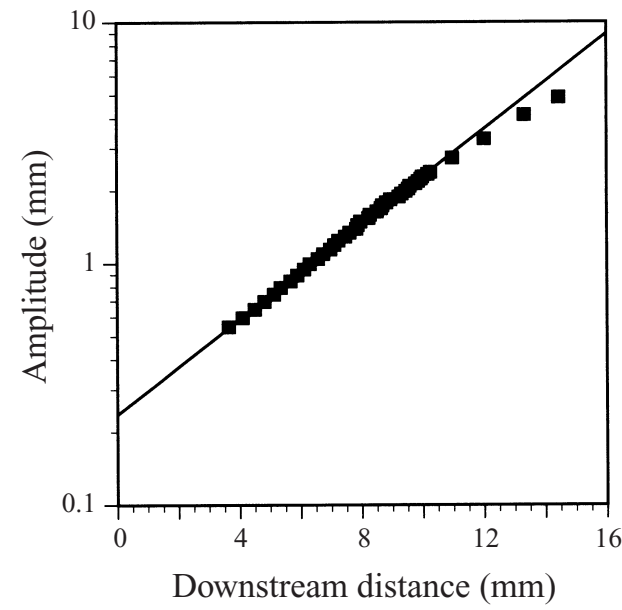

FIGURE 23. Location of the right hand air/water interface of the average image in figure 24 , fitted to an exponential function (straight line in the logarithmic plot).
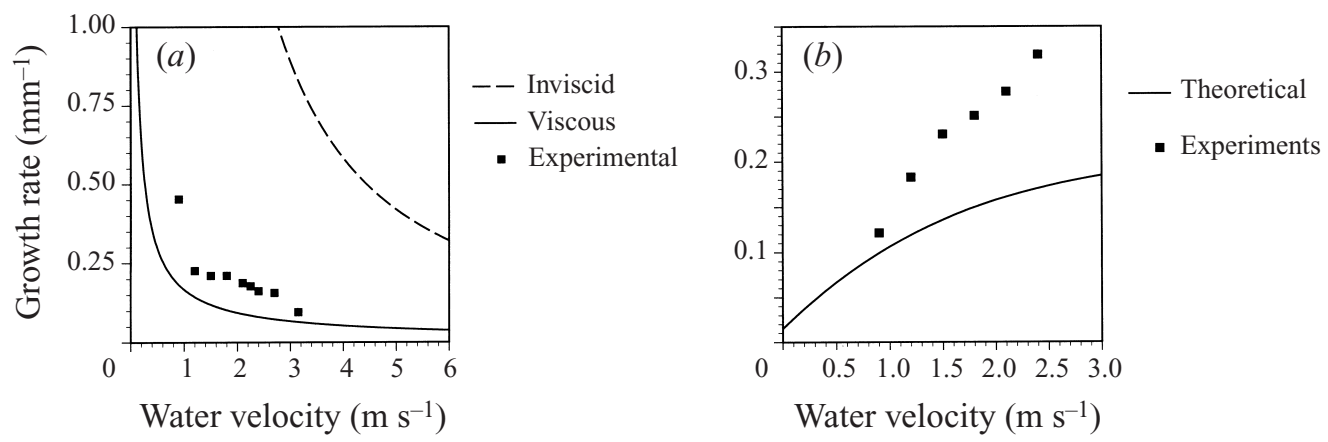

FIGURE 24. Amplitude growth rate of the sinusoidal wave as a function of water velocity for (a) a fixed air velocity $\left(25 \mathrm{~m} \mathrm{~s}^{-1}\right)$, and $(b)$ a fixed $M F R(0.32)$.

complex temporal frequency $\omega$. To enable a comparison with the measured spatial growth coefficient, the values have to be transformed to the form $k_{r}=\omega_{r} / c$ where $c$ is the wave propagation speed.

Some results are summarized in figures $24(a)$ and $24(b)$. For a constant air velocity (figure $24 a$ ), the numerically calculated growth rate decreases with water velocity. Experimentally, the observed trend is similar, but for low water velocities the measurements are less accurate. For high air/water velocity ratios, the air coflow constrains the growth of the sinusoidal waves and the region of linear behaviour is very small. The result is that the spray angle decreases despite the fact that near the nozzle the wave growth rate might be larger. Consequently, most of the spray angle vs. water velocity plots display curves with a maximum (see for example Mansour \& Chigier 1991, or Lozano et al. 1996). In these examples, the spray angle decreases both as the oscillation mode enters either zone $\mathrm{C}$, with the presence of dilatational waves, or zone A, where the air stream dominates over the sheet displacement and spray expansion, reducing the transverse distance over which the droplets can separate from the liquid sheet. For practical purposes, the spray cloud width reaches a maximum for a certain water velocity in zone B. It is curious to observe that the largest spray angle does not necessarily coincide with the maximum amplitude growth rate. 
Figure 24(b) shows the dependence of the amplitude growth on water velocity for a fixed momentum flux ratio MFR. In this situation, the growth rate increases for increasing water velocity (which implies an increase in air velocity as well).

\subsection{Limitations of the linear instability analysis}

For all the variables considered (oscillation frequency, wavelength and oscillation growth rate) results from viscous linear instability analysis seem to correctly describe their qualitative behaviour. It has also been demonstrated for the first time that inclusion of viscous basic velocity profiles greatly improves the predictions when compared with those obtained with the widely used inviscid analysis. For the present measurements, however, the quantitative agreement is not satisfactory. Some considerations are appropriate.

The present linear instability analysis is not capable of properly reproducing the oscillation frequency experimental dependence on sheet thickness. While experimental evidence from several sources indicates that the oscillation frequency scales with the inverse of the sheet thickness, linear instability results are mostly insensitive to these variations. As a consequence, it can be expected that measurements obtained for a thinner sheet will be more closely reproduced via a linear instability analysis. Under some conditions they might even coincide but the agreement should be considered circumstantial.

Further analysis has been performed, considering also different thickness values for the viscous boundary layer in the water stream. A parabolic profile has been assumed for the water boundary layer, with a flat profile for the inner sheet region. When a boundary layer thickness of $h / 10$ is considered, the oscillation frequencies decrease approximately by $20 \%$. Although the reduction is significant, it still does not match the experimental values. Furthermore, the dependence on sheet thickness when this profile is used is also incorrect.

Apparently the effect of water inertia is not correctly captured. This would also explain the discrepancy between numerical and experimental results in the water velocity values for which the maximum frequencies are attained observed in figure 13 . The effect of the air stream seems to be properly described. Given that the general problem seems correctly stated in its mathematical formulation, the discrepancies should be attributed to the linearization of the equations.

\section{Conclusions}

A combined numerical and experimental study has been performed on an airblasted water sheet. Measurements of the oscillation frequency of the longitudinal perturbation, wavelength, and amplitude growth have been obtained. From a linear instability analysis, values for all these parameters have been calculated, for the same flow geometry and conditions as those in the experiments. In this way, a direct comparison between measurements and predictions has been possible. As a novel contribution, viscosity in both air and water flows has been considered in the numerical analysis. The local air boundary layer has been characterized by its thickness $\delta$. This inclusion has been demonstrated to be essential even if a correct qualitative prediction of the parameters mentioned is intended. The increment of the air boundary layer thickness acts to damp the oscillations of the liquid sheet and causes a reduction in the oscillation frequency, the growth rate, as well as in the wavenumber for which the growth is maximum.

It has been observed in the frequency measurements, that for different air velocities, 
a local maximum is obtained for a constant $M F R$ of 0.5 when varying the water velocity. Air and water velocities should be selected accordingly to match this value in order to achieve the fastest atomization. For a fixed water exit velocity, the oscillation frequency increases linearly with air speed, in good agreement with previous studies. This behaviour has been reproduced in the numerical analysis; this is quite remarkable, because, if viscosity is neglected, the predicted dependence is quadratic. It can be concluded that the oscillation cannot be exclusively explained on the grounds of an inviscid Kelvin-Helmholtz instability driven by pressure fluctuations. Again, neglecting viscosity can lead to incorrect predictions.

The results indicate that air velocity mostly determines the oscillation frequency, through air/water interaction near the nozzle exit. Once the oscillation is forced, the amplitude quickly grows when the sheet starts to flap due to the coupling of Kelvin-Helmholtz-type instabilities, affected by viscous effects, on both interfaces. This explains why sinusoidal waves are dominant over dilatational ones for a wide range of $M F R$ values.

For different air and water exit velocities the droplet Sauter mean diameter (SMD) has been calculated. SMD tends to decrease for increasing air velocities. For a fixed air velocity and varying the water speed the $S M D$ curves present a minimum that roughly coincides with the sheet oscillation frequency maximum. This inverse correlation has lead to a non-dimensional plot of $\left(S M D f \mu_{2}\right) / \sigma$ as a function of $M F R$. This empirical relation could be used to predict droplet diameters, but it has to be noted that the measurements have been taken at a distance from the nozzle appropriate to describe the results of primary breakup. After drop formation, secondary breakup and coalescence phenomena can occur, altering the droplet size distribution.

Measurements of the wavelength together with the measured frequencies indicate that the longitudinal waves propagate approximately with the local water velocity, and not with the average of the air and water free-stream velocities as suggested in some papers. As a result, the wavelength increases with downstream distance. The numerical study concludes that the propagation speed is higher than the water exit velocity for those situations where the air accelerating effect on the sheet is noticeable. This happens for high air/water velocity ratios.

The amplitude growth rate has been measured and calculated, and shows a decreasing trend with increasing water velocity, while keeping the air velocity constant. This result contrasts with previously published measurements of spray angle that present curves with a maximum for these velocity values. The discrepancy occurs for the region of high air/water velocity ratios, where the growth is larger, but the reported angles are smaller. This can be explained because, under these circumstances, the region of exponential growth is very small, and the waves are quickly deformed and constrained in their amplitude by the air streams.

As a global conclusion, linear instability predictions of sheet oscillation frequencies, wavelengths and oscillation growth rates improve when viscosity is included in the analysis. Inviscid results are consistently worse in all cases. However, even when viscosity is considered, quantitative agreement is not satisfactory, indicating that the real phenomenon is more complex and probably beyond the capabilities of this type of analysis. Qualitative trends are, in general, correct, but caution has to be exercised when using this method to predict, for example, droplet size distributions. However, it has to be kept in mind that the actual atomization is an inherently threedimensional process, with active participation of transverse waves and longitudinal filaments. Droplet size predictions solely based on longitudinal perturbations cannot 
be strongly based on physical grounds, unless a mechanism is discovered relating these perturbations to the three-dimensional observed effects.

This project has been partially supported by the Comisión Interministerial de Ciencia y Tecnología of the Spanish Government under contract AMB96-0427-C0301, and the Dirección General de Estudios Superiores under contracts PB96-0739C03-03 and BFM2000-1328. The authors want to thank the referees for their insightful comments.

\section{REFERENCES}

Arai, T. \& Hashimoto, H. 1985 Disintegration of a thin liquid sheet in a concurrent gas stream. Proc. 3rd Intl Conf. on Liquid Atomization and Spray Systems, ICLASS-85, London.

BARRERAS, F. 1998 Experimental study of the break-up and atomization of a liquid sheet. $\mathrm{PhD}$ Dissertation, University of Zaragoza (in Spanish).

Benjamin, T. B. 1959 Shearing flow over a wavy boundary. J. Fluid Mech. 6, 161-205.

Birkhoff, G. 1962 Helmholtz and Taylor Instability. Proc. Symp on Applied Mathematics, XII, pp. 55-76. AMS.

Brackmann, U. 1994 Lambdachrome Laser Dyes, pp. 160-161. Lambda Physik GmbH, Göttingen, Germany.

Cousin, J. \& Dumouchel, C. 1996 Effect of viscosity on linear instability of a flat liquid sheet. Atomization Sprays 6, 563-576

Crapper, G. D., Dombrowski, N. \& Jepson, W. P. $1975 a$ Waves on thin sheets of non-Newtonian liquids. Proc. R. Soc. Lond. A 342, 225-236.

Crapper, G. D., Dombrowski, N. \& Pyott, G. $1975 b$ Large amplitude Kelvin-Helmholtz waves on thin liquid sheets. Proc. R. Soc. Lond. A 342, 209-224.

Criminale, W. O., Jackson, T. L., Lasseigne, D. G., \& Joslin, R. D. 1997 Perturbation dynamics in viscous channel flows. J. Fluid Mech. 339, 55-75.

De Kee, D. \& Wissburn, K. S. 1998 Polymer rheology. Physics Today 51, 24-29.

Dombrowski, N., Hasson, D. \& WARD, D. E. 1960 Some aspects of liquid flow through fan spray nozzles. Chem. Engng Sci. 12, 35-50.

Dombrowski, N. \& Johns, W. R. 1963 The aerodynamic instability and disintegration of viscous liquid sheets. Chem. Engng Sci. 18, 203-214.

EAton, J. K. \& Johnston, J. P. 1980 Turbulent flow Re-attachment: an experimental study of the flow and structure behind a backward-facing step. Stanford University, Rep. MD-39.

Eroglu, H. \& Chigier, N. 1991 Liquid sheet instability in a co-flowing air stream. Proc. 5th Intl Conf. on Liquid Atomization and Spray Systems, ICLASS-91, Gaitersburg. MD, USA.

Fraser, R. P., Dombrowski, N. \& Routley, J. H. 1963 The atomization of a liquid sheet by an impinging air stream. Chem. Engng Sci. 18, 339-353.

Gottlieb, D. \& Orszag, S. A. 1977 Numerical Analysis of Spectral Methods: Theory and Applications. CBMS-NSF Regional Conference Series in Applied Mathematics, vol. 26. SIAM.

Hagerty, W. W. \& Shea, J. F. 1955 A study of the stability of plane fluid sheets. Trans. ASME: J. Appl. Mech. December, 509-514.

Ibrahim, E. A. 1994 Spatial instability of a viscous liquid sheets. AIAA Paper 94-0562, pp. 1-7.

Ibrahim, E. A. 1995 Effects of compressibility on the instability of liquid sheets. AIAA Paper 95-2431.

Ibrahim, E. A. \& Akpan, E. T. 1996 Three-dimensional instability of viscous liquid sheets. Atomization Sprays 6, 649-665.

Lefebvre, A. H. 1989 Atomization and Sprays. Hemisphere.

LI, X. \& TAnkin, R. S. 1991 On the temporal instability of a two-dimensional viscous liquid sheet. J. Fluid Mech. 226, 425-443.

Lin, S. P., Lian, Z. W. \& Creighton, B. J. 1990 Absolute and convective instability of a liquid sheet. J. Fluid Mech. 220, 673-689.

Lozano, A., Call, C. J., Dopazo, C. \& García-Olivares, A. 1996 An experimental and numerical study of the atomization of a planar liquid sheet. Atomization Sprays 6, 77-94. 
Lozano, A., García-Olivares, A. \& Dopazo, C. 1998 The instability growth leading to a liquid sheet. Phys. Fluids 10, 2188-2197.

Mansour, A. \& Chigier, N. 1990 Disintegration of liquid sheets. Phys. Fluids A 2, 706-719.

Mansour, A. \& Chigier, N. 1991 Dynamic behavior of liquid sheets. Phys. Fluids A 3, 2971-2980.

Miesen, R. \& Bö̈rsmA, B. J. 1995 Hydrodynamic stability of a sheared liquid film. J. Fluid Mech. 301, 175-202.

Miles, J. W. 1962 On the generation of surface waves by shear flows. Part 4. J. Fluid Mech. 13, 433-488.

OrsZaG, S. A. 1971 Accurate solution of the Orr-Sommerfeld stability equation. J. Fluid Mech. 50, 689-703.

Rangel, R. H. \& Sirignano, W. A. 1991 The linear and non-linear shear instability of a fluid sheet. Phys. Fluids A 3, 2392-2400.

Renardy, Y. 1987 Viscosity and density stratification in vertical Poiseuille flow. Phys. Fluids 30, $1638-1648$.

RizK, N. K. \& Lefebvre, A. H. 1980 The influence of liquid film thickness on airblast atomization. J. Engng Power 102, 706-710.

Savart, F. 1833 Memoire sur le choc d'une veine liquide lancée contre un plan circulaire. Ann. Chim. 54, 56-87

Shavit, U. \& Chigier, N. 1995 The role of dynamic surface tension in air assist atomization. Phys. Fluids 7, 24-33.

Smith, M. \& Davis, S. 1982 The instability of sheared liquid layers. J. Fluid Mech. 121, 187-206.

SQuire, H. B. 1953 Investigation of the instability of moving liquid film. Brit. J. Appl. Phys. 4, 167-169.

StapPer, B. E. \& SAmuelsen, G. S. 1990 An experimental study of the breakup of a two-dimensional liquid sheet in the presence of co-flow air shear. AIAA Paper 89-0461.

Stapper, B. E., Sowa, W. A., \& Samuelsen, G. S. 1992 An experimental study of the effects of liquid properties on the breakup of a two-dimensional liquid sheet. J. Engng Gas Turbine 114, $39-45$.

TAYLOR, G. I. $1959 a$ The dynamics of thin sheets of fluid. I. Water bells. Proc. R. Soc. Lond. A 253 280-296.

TAYLOR, G. I. $1959 b$ The dynamics of thin sheets of fluid. II. Waves on fluid sheets. Proc. R. Soc. Lond. A 253, 296-312.

Teng, C. H., Lin, S. P. \& Chen, J. N. 1997 Absolute and convective instability of a viscous liquid curtain in a viscous gas. J. Fluid Mech. 332, 105-120.

Vich, G., Dumouchel, C. \& Ledoux, M. 1996 Mechanisms of disintegration of flat liquid sheets. 12th Ann. Conf. ILASS-Europe on Liquid Atomization and Spray Systems, Lund, Sweden, June 19-21 (ed. C. J. Bolinder \& L. Fuchs).

YANG, H. Q. 1992 Interfacial instability between a liquid sheet and the surrounding compressible gas. AIAA Paper 92-0461.

YIH, C. S. 1990 Wave formation on a liquid layer for de-icing airplane wings. J. Fluid Mech. 212, $41-53$.

York, J. L., Stubbs, H. E. \& Tek, M. R. 1953 The mechanisms of disintegration of liquid sheets. Trans. ASME October, 1279-1286. 